\title{
Gross-Stark units and $p$-adic iterated integrals attached to modular forms of weight one
}

\author{
Henri Darmon ${ }^{1}$ - Alan Lauder ${ }^{2}$ - Victor Rotger ${ }^{3}$ \\ To Glenn Stevens on his 60th birthday
}

Received: 24 December 2014 / Accepted: 27 October 2015

(C) Fondation Carl-Herz and Springer International Publishing Switzerland 2015

\begin{abstract}
This article can be read as a companion and sequel to the authors' earlier article on Stark points and $p$-adic iterated integrals attached to modular forms of weight one, which proposes a conjectural expression for the so-called $p$-adic iterated integrals attached to a triple $(f, g, h)$ of classical eigenforms of weights $(2,1,1)$. When $f$ is a cusp form, this expression involves the $p$-adic logarithms of so-called Stark points: distinguished points on the modular abelian variety attached to $f$, defined over the number field cut out by the Artin representations attached to $g$ and $h$. The goal of this paper is to formulate an analogous conjecture when $f$ is a weight two Eisenstein series rather than a cusp form. The resulting formula involves the $p$-adic logarithms of units and $p$-units in suitable number fields, and can be seen as a new variant of Gross's $p$-adic analogue of Stark's conjecture on Artin $L$-series at $s=0$.
\end{abstract}

Keywords Eisenstein series $\cdot p$-adic modular forms $\cdot p$-adic iterated integrals $\cdot$ Gross-Stark units

Résumé Cet article peut se lire comme un supplément à l'article des mêmes auteurs sur les "points de Stark" et les intégrales itérées $p$-adiques. Dans cet article antérieur, il est conjecturé que les intégrales itérées $p$-adiques associées à un triplet $(f, g, h)$ de formes modulaires de poids $(2,1,1)$ s'expriment au moyen de points de Stark sur la variété abélienne associée à $f$, définis sur le corps de nombres découpé par le produit tensoriel des représentations d'Artin

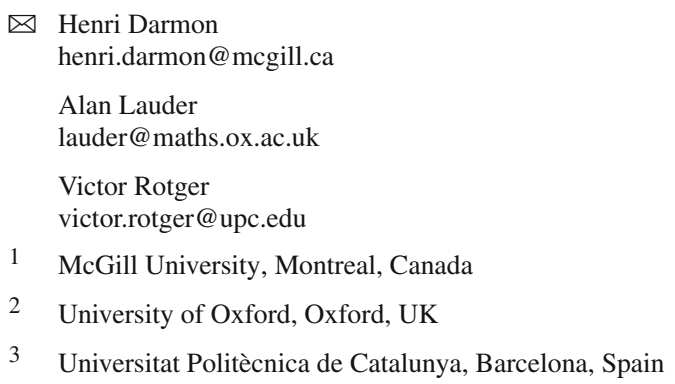


associées à $g$ et à $h$, lorsque $f$ est cuspidale. Le but premier de cet article est d'énoncer une conjecture du même genre quand $f$ provient plutôt d'une série d'Eisenstein. Cette conjecture fait intervenir des logarithmes $p$-adiques d'unités de Stark et de $p$-unités de Gross-Stark, et peut donc être envisagée comme une nouvelle variante des conjectures de Gross étendant au cadre $p$-adique les conjectures de Stark sur les valeurs des fonctions $L$ d'Artin en $s=0$.

\section{Mathematics Subject Classification Primary 11G16, Secondary 11G05 · 11G40}

\section{Contents}

0 Introduction

1 The conjecture

2 Group-theoretic preliminaries

3 Circular units and Eisenstein series of weight one .

4 Elliptic units and theta series of imaginary quadratic fields

4.1 Theoretical evidence

5 Gross-Stark units and theta series of real quadratic fields

6 Exotic forms

References

\section{Introduction}

This article can (and probably should) be read as a companion and sequel to [11], which describes an arithmetic formula for the so-called $p$-adic iterated integrals attached to a triple $(f, g, h)$ of classical modular eigenforms of weights $(2,1,1)$. In the setting considered in [11], where $f$ is assumed to be a cusp form, this formula involves the $p$-adic formal group logarithms of global points on the modular abelian variety $A_{f}$ attached to $f$ by the EichlerShimura construction. The points that arise are defined over the number field cut out by the tensor product $\varrho_{g} \otimes \varrho_{h}$ of the two Artin representations associated to $g$ and $h$. The $p$-adic iterated integral of [11] can be understood as a $p$-adic avatar of the second derivative of the Hasse-Weil-Artin $L$-series $L\left(A_{f}, \varrho_{g} \otimes \varrho_{h}, s\right)$ at its central point $s=1$, and the main conjecture of loc.cit. can therefore be viewed as an analogue (in the $p$-adic setting) of Stark's conjecture on units [21], with unit groups replaced by Mordell-Weil groups of elliptic curves or more general modular abelian varieties.

The goal of the present work is to bring the analogy with Stark's conjecture into sharper focus by studying the iterated integrals of [11] when $f$ is an Eisenstein series of weight 2. The main conjecture of Sect. 1 below transposes the "Elliptic Stark conjecture" of loc.cit. to this setting, essentially by replacing points on elliptic curves with units or $p$-units in number fields having similar Galois-theoretic properties. When $g$ and $h$ are Eisenstein series or theta series attached to a common imaginary quadratic field in which the prime $p$ splits, Sects. 3 and 4 explain how the $p$-adic Stark conjecture can frequently be reduced to classical relations between the Kubota-Leopoldt or Katz $p$-adic $L$-functions and $p$-adic logarithms of circular or elliptic units. To make up for the lack of theoretical evidence in other settings, several numerical verifications of the remaining cases are described in Sects. 5 and 6.

This article owes a lot to the vision and insights of Glenn Stevens, who in several works (notably, [22-24]) exploits the rich interplay between the arithmetic properties of cusp forms and Eisenstein series via their connection with irreducible and reducible two-dimensional Galois representations. Around thirteen years ago, Glenn encouraged the first author to revisit 
his construction of Stark-Heegner points attached to "real quadratic cycles on $\mathcal{H}_{p} \times \mathcal{H}^{\text {" [7] }}$ by replacing the cusp form that arises in it with an Eisenstein series. This led to a refinement [8] of the $p$-adic Gross-Stark units in ring class fields of real quadratic fields (These $p$-units appear in a new guise in Sect. 5, where they play the same role as the Stark-Heegner points of [7] in Section 4 of [11]). ${ }^{1}$ Likewise, but in a purely archimedean setting, the construction [10] of Stark-Heegner points attached to ATR cycles on Hilbert modular varieties gives rise to the geometric construction [6] of Stark units in abelian extensions of ATR extensions of a totally real field from periods of Hilbert modular Eisenstein series. To the extent that the present work bears the same relation to [11] as do [8] and [6] to [7] and [10] respectively, Glenn's suggestion continues to resonate through the years. In the reverse direction, the strategy of substituting elliptic cusp forms for the weight two Eisenstein series that arise in the seminal work of Kato on the Birch and Swinnerton-Dyer conjecture has inspired the general program of studying the arithmetic of elliptic curves based on replacing Beilinson elements attached to a pair of modular units with Beilinson-Flach elements [3,4] and generalised Gross-Schoen diagonal cycles [13,14] (see [1] for an overview of this circle of ideas). With profound gratitude for the influence he has exerted on the authors' mathematical outlook, it is a great pleasure to dedicate this article to Glenn Stevens on the occasion of his 60th birthday.

\section{The conjecture}

Following the notations of [11] which will be adopted throughout, let $M_{k}(N, \chi)_{L}$ and $S_{k}(N, \chi)_{L}$ denote the spaces of modular forms and cusp forms respectively of weight $k$, level $N$, and character $\chi$ with Fourier coefficients in a field $L$. When $L=\mathbb{C}$, it shall be suppressed from the notation.

Let

$$
g \in M_{1}\left(N_{g}, \chi_{g}\right), \quad h \in M_{1}\left(N_{h}, \chi_{h}\right)
$$

be classical eigenforms of weight one. A crucial self-duality assumption made in [11] required the even Dirichlet character $\chi_{g h}:=\chi_{g} \chi_{h}$ to be trivial, and thus the tensor product

$$
\varrho_{g h}:=\varrho_{g} \otimes \varrho_{h}
$$

of the Artin representations attached to $g$ and $h$ to be isomorphic to its contragredient. One of the novelties of the Eisenstein series setting is that this self-duality assumption is not necessary anymore, and can therefore be relaxed: the character $\chi_{g h}$ can now be an arbitrary Dirichlet character, of conductor dividing $N_{g h}:=\operatorname{lcm}\left(N_{g}, N_{h}\right)$. Fix a prime $p \nmid N_{g h}$ and let

$$
\begin{aligned}
& X^{2}-a_{p}(g) X+\chi_{g}(p)=\left(X-\alpha_{g}\right)\left(X-\beta_{g}\right), \\
& X^{2}-a_{p}(h) X+\chi_{h}(p)=\left(X-\alpha_{h}\right)\left(X-\beta_{h}\right)
\end{aligned}
$$

be the $p$-th Hecke polynomials of $g$ and $h$ in $\mathbb{C}[X]$.

It will be convenient to fix a finite extension $L \subset \mathbb{C}$ of $\mathbb{Q}$ over which the representations $\varrho_{g}$ and $\varrho_{h}$ (and hence also $\varrho_{g h}$ ) can be realised. Such an $L$ contains the field generated by the traces of these representations, and can always be chosen to be contained in a cyclotomic field. By enlarging $L$ if necessary, we also assume it contains the roots of unity $\alpha_{g}, \beta_{g}, \alpha_{h}$ and $\beta_{h}$.

\footnotetext{
1 As another illustration of Glenn's influence, let us mention the decisive impact, described in [12], of his theory of overconvergent modular symbols for the efficient polynomial time calculation of the Stark-Heegner points of [7].
} 
Let $g_{\alpha}(z)=g(z)-\beta_{g} g(p z)$ and $g_{\beta}(z)=g(z)-\alpha_{g} g(p z) \in S_{1}\left(N_{g} p, \chi_{g}\right)_{L}$ denote the $p$-stabilizations of $g$ on which the Hecke operator $U_{p}$ acts with eigenvalue $\alpha_{g}$ (resp. $\beta_{g}$ ).

Let

$$
f:=E_{2}\left(1, \chi_{g h}^{-1}\right) \in M_{2}\left(N_{g h}, \chi_{g h}^{-1}\right)
$$

be the weight two Eisenstein series with fourier expansion given by

$$
f(q):=c_{0}+\sum_{n=1}^{\infty}\left(\sum_{d \mid n} \chi_{g h}^{-1}(d) d\right) q^{n} .
$$

The product of the three nebentypus characters attached to the triple $(f, g, h)$ of modular forms of weights $(2,1,1)$ is trivial, and one can define the $p$-adic iterated integral attached to this triple by letting

$$
F:=d^{-1} f=E_{0}^{[p]}\left(\chi_{g h}^{-1}, 1\right)
$$

be the overconvergent Eisenstein series of weight zero attached to the pair $\left(\chi_{g h}^{-1}, 1\right)$ of Dirichlet characters, with Fourier expansion given by

$$
F(q):=\sum_{p \nmid n}\left(\sum_{d \mid n} \chi_{g h}^{-1}(n / d) d^{-1}\right) q^{n},
$$

and considering the modular form

$$
\Xi\left(g_{\alpha}, h\right)=e_{g_{\alpha}^{*}} e_{\text {ord }}(F h),
$$

where

$$
e_{\mathrm{ord}}: M_{1}^{\mathrm{oc}}\left(N_{g h}, \chi_{g}^{-1}\right) \longrightarrow M_{1}^{\mathrm{oc}}\left(N_{g h}, \chi_{g}^{-1}\right)
$$

denotes Hida's ordinary projection on the space $M_{1}\left(N_{g h}, \chi_{g}^{-1}\right)$ of overconvergent modular forms of weight one, and

$$
e_{g_{\alpha}^{*}}: M_{1}^{\mathrm{oc}}\left(N_{g h}, \chi_{g}^{-1}\right) \longrightarrow M_{1}^{\mathrm{oc}}\left(N_{g h}, \chi_{g}^{-1}\right)\left[\left[g_{\alpha}^{*}\right]\right]
$$

denotes the Hecke-equivariant projection to the generalised eigenspace attached to the system of Hecke eigenvalues for the dual form $g_{\alpha}^{*}$ of $g_{\alpha}$. The modular form $\Xi\left(g_{\alpha}, h\right)$ is overconvergent and ordinary, but need not be classical, since the conclusion of Coleman's classicality theorem fails in general in weight one. For this reason, it is convenient to assume that Hypothesis C of [11], referred to there as the "classicality property", holds for $g_{\alpha}$. This hypothesis stipulates that the natural inclusion

$$
M_{1}\left(N p, \chi_{g}^{-1}\right) \mathbb{C}_{p}\left[g_{\alpha}^{*}\right] \hookrightarrow M_{1}^{\text {oc, ord }}\left(N, \chi_{g}^{-1}\right)\left[\left[g_{\alpha}^{*}\right]\right]
$$

of the eigenspace attached to $g_{\alpha}$ (consisting entirely of classical forms) into the associated generalised eigenspace in the space of $p$-adic modular forms, maps $M_{1}\left(N p, \chi_{g}^{-1}\right)_{\mathbb{C}_{p}}\left[g_{\alpha}^{*}\right]$ into the generalized eigenspace $S_{1}^{\text {oc,ord }}\left(N, \chi_{g}^{-1}\right)\left[\left[g_{\alpha}^{*}\right]\right]$ of $p$-adic cusp forms and gives rise to an isomorphism

$$
M_{1}\left(N p, \chi_{g}^{-1}\right)_{\mathbb{C}_{p}}\left[g_{\alpha}^{*}\right] \simeq S_{1}^{\mathrm{oc}, \text { ord }}\left(N, \chi_{g}^{-1}\right)\left[\left[g_{\alpha}^{*}\right]\right]
$$

between these two $\mathbb{C}_{p}$-vector spaces. 
When $g$ is cuspidal, it shall be assumed throughout that $\alpha_{g} \neq \beta_{g}$, i.e., that $g$ is regular at $p$, and that $g$ is not a theta series of a real quadratic field in which the prime $p$ is split. As explained in [11, Proposition 1.2], the main result of [5] then implies the classicality property for $g_{\alpha}$.

On the other hand, when $g=E_{1}\left(\chi^{+}, \chi^{-}\right)$is the Eisenstein series associated to a pair of Dirichlet characters $\chi^{+}$and $\chi^{-}$, the space $M_{1}\left(N p, \chi_{g}^{-1}\right)_{\mathbb{C}_{p}}\left[g_{\alpha}^{*}\right]$ maps into $S_{1}^{\text {oc,ord }}\left(N, \chi_{g}^{-1}\right)\left[\left[g_{\alpha}^{*}\right]\right]$ if and only if $\chi^{+}(p)=\chi^{-}(p)$, that is to say, if and only if $g$ is not regular at $p$ (cf. [11, Proposition 1.3]), and non-regularity is therefore a necessary condition for the classicality property to be satisfied.

Given an element $\gamma$ in the $L$-linear dual space $M_{1}\left(N_{g h}, \chi_{g}^{-1}\right)_{L}\left[g_{\alpha}^{*}\right]^{\vee}$, the $p$-adic iterated integral attached to the pair $(\gamma, h)$ is defined to be

$$
\int_{\gamma} f \cdot h:=\gamma\left(e_{g_{\alpha}^{*}} e_{\mathrm{ord}}(F h)\right)=\gamma\left(\Xi\left(g_{\alpha}, h\right)\right) .
$$

Our goal is to study this $p$-adic iterated integral: more precisely, to understand when the classical modular form $\Xi\left(g_{\alpha}, h\right)$ with $\mathbb{C}_{p}$ coefficients is non-trivial, and, when it is, give an arithmetic interpretation for its position in $M_{1}\left(N_{g h}, \chi_{g}^{-1}\right)_{\mathbb{C}_{p}}\left[g_{\alpha}^{*}\right]$ relative to the natural $L$-rational structure on this $\mathbb{C}_{p}$-vector space given by $M_{1}\left(N_{g h}, \chi_{g}^{-1}\right)_{L}\left[g_{\alpha}^{*}\right]$.

As in [11], the four-dimensional tensor product representation

$$
\varrho_{g h}:=\varrho_{g} \otimes \varrho_{h}
$$

plays a key role in this arithmetic interpretation. Let $H$ be the smallest number field through which $\varrho_{g h}$ factors, and set

$$
U_{g h}:=L \otimes \mathcal{O}_{H}^{\times}, \quad U_{g h}^{(p)}:=L \otimes\left(\mathcal{O}_{H}[1 / p]^{\times} / p^{\mathbb{Z}}\right),
$$

viewed as finite-dimensional $L$-linear representations of $G_{\mathbb{Q}}$.

Lemma 1.1 Let s denote the multiplicity of the trivial representation in $\varrho_{g h}$, and let

$$
d_{g h}:=\operatorname{dim}_{L} \operatorname{hom}_{G_{\mathbb{Q}}}\left(\varrho_{g h}, U_{g h}\right), \quad d_{g h}^{(p)}:=\operatorname{dim}_{L} \operatorname{hom}_{G_{\mathbb{Q}}}\left(\varrho_{g h}, U_{g h}^{(p)}\right) .
$$

Then

(1) $d_{g h}=2-s$,

(2) $d_{g h}^{(p)}=2+\operatorname{dim}_{L} \varrho_{g h}^{G_{\mathbb{Q} p}}-2 s$.

Proof This follows from Herbrand's Galois-equivariant strengthening of Dirichlet's $S$-unit theorem, combined with Frobenius reciprocity. See for instance [25, Ch.I, §3, §4] or [15, Prop. 3.2.2].

In particular, when $s=0$, Lemma 1.1 shows that $d_{g h}=2$ and that the same is true for $d_{g h}^{(p)}$ when $\varrho_{g h}$ admits no $G_{\mathbb{Q}_{p}}$-invariants. Furthermore, it is not hard to see that $d_{g h}^{(p)} \geq 2$ when at least one of $g$ or $h$ is a cusp form. This is in marked contrast with the setting explored in [11] where $U_{g h}$ and $U_{g h}^{(p)}$ were replaced by the Mordell-Weil group $E(H)$ of an elliptic curve $E$, tensored with $L$ : in this case no simple elementary formula was available for the analogues of $d_{g h}$ and $d_{g h}^{(p)}$, and these quantities appear to be 0 quite often. This is why the necessity of the "vanishing hypothesis" (Hypothesis B) arose in [11], whereas here it is unecessary.

Fix an embedding of $H$ into $\mathbb{C}_{p}$. This equips $H$, and hence also $U_{g h}$ and $U_{g h}^{(p)}$, with natural actions of $G_{\mathbb{Q}_{p}}$. Since $p$ is assumed to be prime to $N_{g h}$, the action of $G_{\mathbb{Q}_{p}}$ on $H$ is unramified, 
and therefore the spaces $U_{g h}$ and $U_{g h}^{(p)}$ inherit a natural linear action of the Frobenius operator $\sigma_{p}$, which acts on $\varrho_{g h}$ with eigenvalues $\alpha_{g} \alpha_{h}, \alpha_{g} \beta_{h}, \beta_{g} \alpha_{h}$, and $\beta_{g} \beta_{h}$. Write

$$
\varrho_{g}:=\varrho_{g}^{\alpha_{g}} \oplus \varrho_{g}^{\beta_{g}}, \quad \varrho_{h}:=\varrho_{h}^{\alpha_{h}} \oplus \varrho_{h}^{\beta_{h}}
$$

for the decompositions of $\varrho_{g}$ and $\varrho_{h}$ into one-dimensional frobenius eigenspaces, when these are defined, i.e., when $\sigma_{p}$ acts on the relevant representation with distinct eigenvalues. When $g$ is cuspidal, so that $\alpha_{g} \neq \beta_{g}$ by our running assumption, we attach to $g_{\alpha}$ a two-dimensional subspace of the representation $\varrho_{g h}$ by setting

$$
\varrho_{g h}^{g_{\alpha}}:=\varrho_{g}^{\alpha_{g}} \otimes \varrho_{h} .
$$

When $g$ is Eisenstein, we have $\alpha_{g}=\beta_{g}$ and we simply define

$$
\varrho_{g h}^{g_{\alpha}}:=W \otimes \varrho_{h},
$$

where $W$ is any line in $\varrho_{g}$ which is not stable under $G_{\mathbb{Q}}$.

When $d_{g h}^{(p)}=2$, it is possible to associate to the pair $\left(g_{\alpha}, h\right)$ a $p$-adic regulator by choosing an $L$-basis $\left(\Phi_{1}, \Phi_{2}\right)$ for $\operatorname{hom}_{G_{\mathbb{Q}}}\left(\varrho_{g h}, U_{g h}^{(p)}\right)$ and an $L$-basis $\left(v_{1}, v_{2}\right)$ for $\varrho_{g h}^{\alpha_{g}}$ and setting

$$
R_{g_{\alpha}}\left(\varrho_{g h}\right):=\left(\begin{array}{ll}
\log _{p}\left(\Phi_{1}\left(v_{1}\right)\right) & \log _{p}\left(\Phi_{1}\left(v_{2}\right)\right) \\
\log _{p}\left(\Phi_{2}\left(v_{1}\right)\right) & \log _{p}\left(\Phi_{2}\left(v_{2}\right)\right)
\end{array}\right) .
$$

This matrix is well-defined up to left and right multiplication by invertible matrices with entries in $L$, and hence its determinant is well-defined modulo $L^{\times}$. This determinant belongs to $\mathbb{Q}_{p}^{\mathrm{u}-\mathrm{r}} \otimes L$, and $\sigma_{p}$ acts on it with the eigenvalue $\alpha_{g} \alpha_{h} \alpha_{g} \beta_{h}=\alpha_{g} / \beta_{g} \cdot \chi_{g h}(p)$.

Recall the unit $u_{g_{\alpha}}$ in $U_{g h}$ (when $g$ is a cusp form) or in $U_{g h}^{(p)}$ (when $g$ is an Eisenstein series) attached to the $p$-stabilised eigenform $g_{\alpha}$ in $[11, \S 1.2]$. It belongs to the $\operatorname{Ad}^{0}(g)$-isotypic part of $U_{g h}$ (resp. of $U_{g h}^{(p)}$ ), and is an eigenvector for $\sigma_{p}$ with eigenvalue $\alpha_{g} / \beta_{g}$.

Finally, if $\chi$ is a Dirichlet character of conductor $m$, let

$$
\mathfrak{g}(\chi):=\sum_{a=1}^{m} \chi^{-1}(a) e^{2 \pi i a / m}
$$

be the usual Gauss sum. Our main conjecture is:

Conjecture 1.2 If $d_{g h}^{(p)}>2$, then the modular form $\Xi\left(g_{\alpha}, h\right)$ is 0 . Otherwise,

$$
\Xi\left(g_{\alpha}, h\right) \text { belongs to } \Omega_{g h} \cdot M_{1}\left(N_{g h}, \chi_{g}^{-1}\right)\left[g_{\alpha}^{*}\right]_{L},
$$

where the period $\Omega_{g h}$ is given by the formula

$$
\Omega_{g h}:=\frac{\operatorname{det}\left(R_{g_{\alpha}}\left(\varrho_{g h}\right)\right)}{\mathfrak{g}\left(\chi_{g h}\right) \log _{p}\left(u_{g_{\alpha}}\right)} .
$$

As discussed in the introduction, this conjecture is directly inspired by the "elliptic Stark conjectures" of [11] which apply to the setting where $f$ is replaced with a weight two cusp form attached to an elliptic curve. Conjecture 1.2 is closer in spirit to the $p$-adic Gross-Stark conjectures, since its regulator term involved units and $p$-units of number fields rather than global points on elliptic curves, but it does not appear to follow from any of the variants of Stark's conjecture previously available in the literature.

The remainder of this paper shall be devoted to providing both theoretical and experimental support for Conjecture 1.2. 


\section{Group-theoretic preliminaries}

This section collects some general representation-theoretic results concerning the structure of the representation $\varrho_{g h}$ appearing in Conjecture 1.2. We refer to [16,20] for generalities on Artin representations and their connection to modular forms of weight one.

Let $V_{g}$ and $V_{h}$ denote two-dimensional $L$-vector spaces which realise $\varrho_{g}$ and $\varrho_{h}$ respectively, and denote by $V_{g h}=V_{g} \otimes_{L} V_{h}$ the $L$-vector space realising $\varrho_{g h}$.

The image of $\varrho_{g}$ is a finite subgroup $G \subset \mathbf{G L}_{2}(\mathbb{C})$. The well-known classification of such groups implies that the natural image $\Gamma$ of $G$ in $\mathbf{P G L}_{2}(\mathbb{C})$ is isomorphic to

(1) a cyclic group, if $g$ is an Eisenstein series;

(2) a dihedral group, if $g$ is the theta series attached to a finite order character of a (real or imaginary) quadratic field;

(3) the group $A_{4}, S_{4}$ or $A_{5}$. In this last case, the form $g$ is said to be exotic.

The group $G$ is then isomorphic to

$$
G=(Z \times \tilde{\Gamma}) /\langle \pm 1\rangle,
$$

where $Z=G \cap \mathbb{C}^{\times}$is the center of $G$, and $\tilde{\Gamma}=G \cap \mathbf{S L}_{2}(\mathbb{C})$ is the universal central extension of $\Gamma$. It is a non-split central extension of $\Gamma$ by the group $\langle \pm 1\rangle \subset Z$, and is called the binary tetrahedral, octahedral, or icosahedral group (of order 24,48 , and 120) when $\Gamma=A_{4}, S_{4}$ and $A_{5}$ respectively. The irreducible representations of $G$ are obtained in a simple way from those of $\tilde{\Gamma}$ : more precisely, if $\varrho_{1}, \ldots \varrho_{t}$ is a complete list of the irreducible representations of $\tilde{\Gamma}$, the irreducible representations of $G$ are simply of the form $\chi_{Z} \otimes \varrho_{j}$, where $\chi_{Z}$ is a character of $Z$ satisfying $\chi_{Z}(-1)=\varrho_{j}(-1)$.

When $\varrho_{g}$ has cyclic projective image, it decomposes as a direct sum of two onedimensional representations attached to Dirichlet characters $\chi_{1}$ and $\chi_{2}$, and $g=E_{1}\left(\chi_{1}, \chi_{2}\right)$ is the weight one Eisenstein series attached to this pair of characters.

When $\varrho_{g}$ (or $\varrho_{h}$ ) has dihedral projective image, it is induced from a one-dimensional character $\psi_{g}: G_{K} \longrightarrow \mathbb{C}^{\times}$of a quadratic field $K$, and $g$ is the theta series attached to this character. The representation

$$
V_{\psi_{g}}:=\operatorname{Ind}_{K}^{\mathbb{Q}}\left(\psi_{g}\right): G_{\mathbb{Q}} \longrightarrow \mathbf{G L}_{2}(\mathbb{C})
$$

becomes reducible when restricted to $K$, where it is isomorphic to $L\left(\psi_{g}\right) \oplus L\left(\psi_{g}^{\prime}\right)$, where $\psi_{g}^{\prime}$ denotes the character obtained by composing $\psi_{g}$ with the automorphism of $K / \mathbb{Q}$. Furthermore, $V_{\psi_{g}}$ is odd if and only if either

(a) $K$ is an imaginary quadratic field, or

(b) $K$ is a real quadratic field, and $\psi_{g}$ is of mixed signature, i.e., it is even at one of the archimedean places of $K$, and odd at the other.

Note that $V_{\psi_{g}}$ is irreducible (and hence, $g$ is a cusp form) precisely when $\psi_{g} \neq \psi_{g}^{\prime}$.

The most basic invariant of the tensor product $V_{g h}$ is its decomposition

$$
V_{g h}=V_{1} \oplus \cdots \oplus V_{t}
$$

into irreducible representations. Write $\underline{d}:=\left(d_{1}, \ldots, d_{t}\right)$ for the dimensions of the $V_{j}$ that arise in this decomposition, arranged by convention in non-increasing order. There are five possibilities for $\underline{d}$, corresponding to the possible partitions of 4 . 
If $g$ and $h$ are both reducible, i.e., correspond to Eisenstein series, then clearly $\underline{d}=$ $(1,1,1,1)$. Assume from now on that at least one of $g$ or $h$ is a cusp form, hence is associated to an irreducible two-dimensional representation.

Lemma 2.1 The representation $V_{g h}$ contains a subrepresentation of dimension 1 if and only if $h$ is a twist of $\bar{g}$, i.e., $V_{h}=V_{g}^{\vee}(\iota)$, for some Dirichlet character $\iota$. In that case,

(1) $\underline{d}=(3,1)$ if and only if $g$ is exotic;

(2) $\underline{d}=(2,1,1)$ if and only if $g$ is dihedral and $\psi_{g}^{-}:=\psi_{g} / \psi_{g}^{\prime}$ is not a quadratic (genus) character;

(3) $\underline{d}=(1,1,1,1)$ if and only if $g$ is dihedral and $\psi_{g}^{-}$is a genus character.

Proof The one-dimensional subrepresentation of $V_{g h}$ is isomorphic to $L(\iota)$ for some Dirichlet character $\iota$ (viewed as a Galois character). This implies that

$$
V_{g h} \otimes L\left(\iota^{-1}\right)=\operatorname{hom}\left(V_{g}^{\vee}(\iota), V_{h}\right)
$$

has a $G_{\mathbb{Q}}$-invariant vector. Since at least one of $V_{g}$ or $V_{h}$ is irreducible, Schur's Lemma implies that $V_{h}$ is isomorphic to $V_{g}^{\vee}(\iota)$. It follows that

$$
V_{g h}=W \oplus L(\iota), \quad \text { where } W:=\operatorname{Ad}^{0}\left(V_{g}\right)(\iota)
$$

is the twist by $\iota$ of the adjoint representation attached to $V_{g}$. Lemma 2.1 now follows by noting that:

(i) if $\varrho_{g}$ has projective image isomorphic to $\Gamma=A_{4}, S_{4}$ or $A_{5}$, then $\operatorname{Ad}^{0}\left(V_{g}\right)$ factors through $\Gamma$ and is a three-dimensional irreducible representation of this group, as can be seen, for instance, by consulting the character tables of $\Gamma$ and $\tilde{\Gamma}$;

(ii) if $\varrho_{g}$ has dihedral projective image, and thus is induced from a character $\psi_{g}$ of a quadratic field $K$, then

$$
\operatorname{Ad}^{0}\left(V_{g}\right)=L\left(\chi_{K}\right) \oplus V_{\psi_{g}^{-}},
$$

where $\chi_{K}$ is the quadratic Dirichlet character attached to $K$.

The next lemma takes up the case where $\underline{d}=(2,2)$.

Lemma 2.2 The representation $V_{g h}$ decomposes as a sum of two irreducible representations of dimension two if and only if:

(1) Exactly one of $g$ or $h$ is a weight one Eisenstein series, say $h=E_{1}\left(\chi_{1}, \chi_{2}\right)$, in which case

$$
V_{g h}=V_{g}\left(\chi_{1}\right) \oplus V_{g}\left(\chi_{2}\right) .
$$

(2) Both $g$ and $h$ are theta series attached to characters $\psi_{g}$ and $\psi_{h}$ of the same quadratic field $K$ for which neither of the characters

$$
\psi_{1}:=\psi_{g} \psi_{h}, \quad \psi_{2}:=\psi_{g} \psi_{h}^{\prime}
$$

is equal to its conjugate over $K$. In this case,

$$
V_{g h}=V_{\psi_{1}} \oplus V_{\psi_{2}} .
$$

The representations $V_{\psi_{1}}$ and $V_{\psi_{2}}$ are non-isomorphic, and if $K$ is real quadratic, they are of opposite (pure) signatures. 
Proof It is clear that $\underline{d}=(2,2)$ when exactly one of $g$ or $h$ is an Eisenstein series. Assume for the rest of the proof that both $g$ and $h$ are cuspidal. Let $H_{g} / \mathbb{Q}$ and $H_{h} / \mathbb{Q}$ be the extensions of $\mathbb{Q}$ cut out by the representations $\varrho_{g}$ and $\varrho_{h}$, let $H:=H_{g} \cap H_{h}$, and let $H_{g h}$ be the compositum of the extensions $H_{g}$ and $H_{h}$, which are linearly disjoint over $H$. Write

$$
\begin{aligned}
G_{g h} & :=\operatorname{Gal}\left(H_{g h} / \mathbb{Q}\right), & G_{g}:=\operatorname{Gal}\left(H_{g} / \mathbb{Q}\right), & G_{h}:=\operatorname{Gal}\left(H_{h} / \mathbb{Q}\right), \\
\mathcal{A}_{g}:=\operatorname{Gal}\left(H_{g} / H\right), & \mathcal{A}_{h}:=\operatorname{Gal}\left(H_{h} / H\right) . & & G_{0}=\operatorname{Gal}(H / \mathbb{Q}) .
\end{aligned}
$$

The resulting field diagram is described below

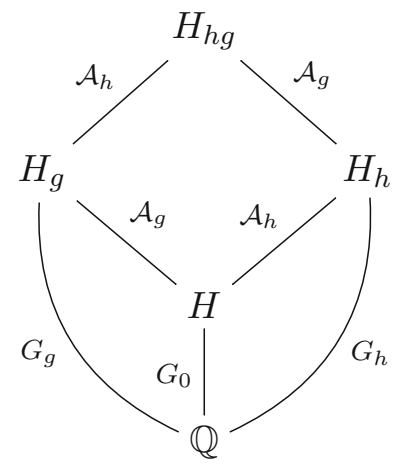

By hypothesis, the group $G_{g}$ (resp. $G_{h}$ ) acts faithfully on $V_{g}$ (resp. $V_{h}$ ).

Observe first that the groups $\mathcal{A}_{g}$ and $\mathcal{A}_{h}$ are necessarily abelian. For if $\mathcal{A}_{g}$ (say) were non-abelian, the two-dimensional vector space $V_{g}$ on which $\mathcal{A}_{g}$ acts faithfully would be irreducible as an $\mathcal{A}_{g}$-module. Since $\mathcal{A}_{g}$ acts trivially on $V_{h}$, the irreducible $\mathcal{A}_{g}$-submodules of $V_{g h}$ would have to all be of the form $V_{g} \otimes L_{h}$ for some one-dimensional $L$-vector subspace $L_{h} \subset V_{h}$. This would force any two-dimensional irreducible sub-representation of $V_{g h}$ to also be of this form. But $V_{h}$ contains no $G_{h}$-stable lines and the vector space $V_{g} \otimes L_{h}$ therefore cannot be stable under the full action of $G_{g h}$.

One can now distinguish two cases:

Case A At least one of $G_{g}$ or $G_{h}$ has a projective image isomorphic to $A_{4}, S_{4}$ or $A_{5}$. Assume without loss of generality that this is the case for $G_{g}$. The only abelian normal subgroups of $\tilde{\Gamma}_{g}$ are contained in its center, and hence, the same is true for the abelian normal subgroups of $G_{g}$. It follows that $\mathcal{A}_{g}$ acts on $V_{g}$ as scalar multiplications, and that $G_{0}$ has a quotient isomorphic to $\Gamma$. Hence the same assertions hold for $G_{h}$ : in other words, both $G_{g}$ and $G_{h}$ are central extensions of a common $\Gamma \subset \mathbf{P G L}_{2}(\mathbb{C})$ which arises as a quotient of $G_{0}$. A study of the character table of $\tilde{\Gamma}$ reveals that the tensor products of two irreducible two-dimensional representations of this group never decomposes as a sum of two-dimensional representations. This rules out the scenario where $\underline{d}=(2,2)$ in Case A.

Case B The groups $G_{g}$ and $G_{h}$ have dihedral projective image. The representation $\varrho_{g}$ is then induced from a character $\psi_{g}$ of a quadratic field $K$, which satisfies $\psi_{g} \neq \psi_{g}^{\prime}$, since $V_{g}$ is irreducible. Hence $V_{g}$ is isomorphic to $L\left(\psi_{g}\right) \oplus L\left(\psi_{g}^{\prime}\right)$ as a $G_{K}$-module, and any element of $G_{\mathbb{Q}}-G_{K}$ interchanges $L\left(\psi_{g}\right)$ and $L\left(\psi_{g}^{\prime}\right)$. In particular, the representation $V_{g h}$ is isomorphic to $V_{h}\left(\psi_{g}\right) \oplus V_{h}\left(\psi_{g}^{\prime}\right)$ as a $G_{K}$-module, and the following cases arise: 
- $V_{h}$ is reducible as a $G_{K}$-module, and hence $\varrho_{h}$ is induced from a character of $K$.

- $V_{h}$ remains irreducible as a $G_{K}$-module. The reducibility of $V_{g h}$ then forces

$$
V_{h}\left(\psi_{g}\right) \simeq V_{h}\left(\psi_{g}^{\prime}\right), \text { hence } V_{h} \simeq V_{h}\left(\psi_{g} / \psi_{g}^{\prime}\right) \text { as } G_{K} \text { - modules. }
$$

For otherwise, the only non-trivial $G_{K}$-stable subspaces of $V_{g h}$ would be $V_{h}\left(\psi_{g}\right)$ and $V_{h}\left(\psi_{g}^{\prime}\right)$, which are not preserved by elements of $G_{\mathbb{Q}}-G_{K}$. Taking determinants on both sides of (6) reveals that $\psi_{g} / \psi_{g}^{\prime}$ is a quadratic (and hence a genus) character of $K$, whose induced representation is a direct sum $\chi_{1} \oplus \chi_{2}$ of two quadratic Dirichlet characters attached to quadratic extensions $K_{1}$ and $K_{2}$ respectively, satisfying $\chi_{1} \chi_{2}=\chi_{K}$, where $\chi_{K}$ is the quadratic character attached to $K$. Inducing (6) from $K$ to $\mathbb{Q}$ shows that

$$
V_{h} \oplus V_{h}\left(\chi_{K}\right) \simeq V_{h}\left(\chi_{1}\right) \oplus V_{h}\left(\chi_{2}\right) \quad \text { as } \quad G_{\mathbb{Q}} \text {-modules. }
$$

Since $V_{h}$ and $V_{h}\left(\chi_{K}\right)$ are assumed to be non-isomorphic, it may be assumed, after eventually interchanging $\chi_{1}$ and $\chi_{2}$, that $V_{h}$ is isomorphic to $V_{h}\left(\chi_{1}\right)$, and therefore, that $V_{h}$ is induced from a character of $K_{1}$. But the same is also true of $V_{g}$, which is a twist of a representation of the dihedral group of order 8 , and as such is simultaneously induced from characters of all three of the quadratic fields $K, K_{1}$ and $K_{2}$.

After replacing $K$ by $K_{1}$ in the second scenario, it follows that $V_{g}$ and $V_{h}$ are induced from characters of the same quadratic field $K$. Writing $\psi_{h}$ for the character of $K$ satisfying $V_{h}=V_{\psi_{h}}$, the representation $V_{g h}$ breaks up as

$$
V_{g h}=V_{\psi_{1}} \oplus V_{\psi_{2}}, \quad \psi_{1}:=\psi_{g} \psi_{h}, \quad \psi_{2}:=\psi_{g} \psi_{h}^{\prime} .
$$

It follows from this that $\psi_{1}$ and $\psi_{2}$ have the same central character, and that $\underline{d}=(2,2)$ precisely when $\psi_{1} \neq \psi_{1}^{\prime}$ and $\psi_{2} \neq \psi_{2}^{\prime}$ (Recall that the central character of a character $\psi$ of $G_{K}$ is the Dirichlet character arising from the restriction to the idéles of $\mathbb{Q}$ of the Hecke character of $K$ associated by class field theory to $\psi$ ). To see that $V_{\psi_{1}}$ and $V_{\psi_{2}}$ are never isomorphic, note that the irreducibility of $V_{h}$ implies that $\psi_{h} \neq \psi_{h}^{\prime}$, and hence that $\psi_{1} \neq \psi_{2}$; and that likewise, the irreducibility of $V_{g}$ implies that $\psi_{1} \neq \psi_{2}^{\prime}$. When $K$ is real quadratic, it is readily checked that, since $\psi_{g}$ and $\psi_{h}$ are both of mixed signature, the ring class characters $\psi_{1}=\psi_{g} \psi_{h}$ and $\psi_{2}=\psi_{g} \psi_{h}^{\prime}$ are of opposite pure signatures. The lemma follows.

The "generic" case where $\underline{d}=(4)$ encompasses all scenarios where the representations $\varrho_{g}$ and $\varrho_{h}$ cut out linearly disjoint extensions of $\mathbb{Q}$. When it is irreducible, the Artin representation $V_{g h}$ occurs with mutiplicity two in $L \otimes \mathcal{O}_{H}^{\times}$and is undistinguished enough to probably never have garnered explicit attention in connection with the Stark or Gross-Stark conjectures. Nonetheless the special cases described in Lemmas 2.3 and 2.4 below are worth singling out.

Suppose that

$$
\varrho_{h}: \operatorname{Gal}\left(H_{h} / \mathbb{Q}\right) \longrightarrow \tilde{\Gamma} \subset \mathbf{G L}_{2}(\mathbb{C})
$$

has projective image $\Gamma=\tilde{\Gamma} / Z \simeq S_{4}$, and that $\varrho_{g}$ is the (non-faithful) two-dimensional representation of $\operatorname{Gal}\left(H_{h} / \mathbb{Q}\right)$ obtained from $\varrho_{h}$ by composing it with the natural sequence of homomorphisms $\tilde{\Gamma} \longrightarrow S_{4} \longrightarrow S_{3} \subset \mathbf{G L}_{2}(\mathbb{C})$. Then $\varrho_{g h}$ can be described concretely in terms of the induced representation of a quadratic character of the quartic subfield $K$ of $H_{h}$ fixed by $S_{3} \subset S_{4}$. More precisely, the natural exact sequence

$$
1 \longrightarrow Z \longrightarrow \operatorname{Gal}\left(H_{h} / K\right) \longrightarrow S_{3} \longrightarrow 1
$$

splits. Since $Z$ is a cyclic group of even cardinality, it follows that $\mathrm{Gal}\left(H_{h} / K\right)$ has three distinct surjective homomorphisms to \pm 1 , of which only one is trivial on $Z$. Let $\eta$ be a 
quadratic character of $\mathrm{Gal}\left(H_{h} / K\right)$ which is non-trivial on $Z$, and let $M$ be the octic extension of $\mathbb{Q}$ cut out by $\eta$, as summarised in the following field diagram:

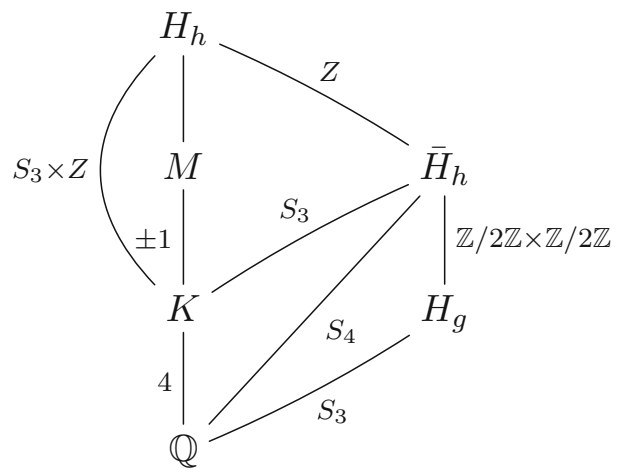

Lemma 2.3 For $\varrho_{g}$ and $\varrho_{h}$ as above,

$$
\varrho_{g h}=\operatorname{Ind}_{K}^{\mathbb{Q}} \eta .
$$

In particular,

$$
\operatorname{hom}\left(V_{g h}, \mathbb{C} \otimes \mathcal{O}_{H}^{\times}\right)=\mathbb{C} \otimes \mathcal{O}_{M, 1}^{\times},
$$

where $\mathcal{O}_{M, 1}^{\times}$denotes the group of units of $M$ whose norm to $K$ is equal to 1 .

Proof The first assertion follows from a direct calculation using the character table of $\tilde{S}_{4}$, and the second from Frobenius reciprocity.

Another simple, concrete instance of an irreducible $V_{g h}$ arises when $K$ is a non-totally real quintic extension of $\mathbb{Q}$ whose Galois closure $\tilde{K}$ has Galois group $A_{5}$ over $\mathbb{Q}$. There are exactly two conjugacy classes of embeddings of $\operatorname{Gal}(\tilde{K} / \mathbb{Q})=A_{5}$ into $\mathbf{P G L}_{2}(\mathbb{C})$; choosing one gives rise to a projective representation

$$
\bar{\varrho}_{g}: G_{\mathbb{Q}} \rightarrow \operatorname{Gal}(\tilde{K} / \mathbb{Q}) \simeq A_{5} \subset \mathbf{P G L}_{2}(\mathbb{C}) .
$$

Tate's lifting theorem produces a linear lift $\varrho_{g}: G_{\mathbb{Q}} \longrightarrow \mathbf{G L}_{2}(\mathbb{C})$ of $\bar{\varrho}_{g}$, which is odd since $K$ is not totally real, and hence corresponds to a modular form $g$. It can further be assumed that the field $H$ cut out by $\varrho_{g}$ is a cyclic extension of $\tilde{K}$ of 2-power degree, so that $\varrho_{g}(\operatorname{Gal}(H / \tilde{K}))$ consists of scalar matrices whose diagonal entries are 2-power roots of unity. Let $\sigma$ be an automorphism of $\mathbb{C}$ which agrees with complex conjugation on the 2-power roots of unity and sends $\sqrt{5}$ to $-\sqrt{5}$, and let $h$ be the modular form obtained by applying $\sigma$ to $g$.

Lemma 2.4 For $\varrho_{g}$ and $\varrho_{h}$ as above,

$$
V_{g h} \oplus \mathbb{C}=V_{5},
$$

where $V_{5}$ is the natural permutation representation of $A_{5}$ acting on 5 letters. In particular, $\operatorname{hom}\left(V_{g h}, \mathbb{C} \otimes \mathcal{O}_{H}^{\times}\right)=\mathbb{C} \otimes \mathcal{O}_{K}^{\times}, \quad \operatorname{hom}\left(V_{g h}, \mathbb{C} \otimes \mathcal{O}_{H}[1 / p]^{\times}\right)=\mathbb{C} \otimes\left(\mathcal{O}_{K}[1 / p]^{\times} / p^{\mathbb{Z}}\right)$.

Proof See for instance the proof of Thm. 6.9 of [14]. 


\section{Circular units and Eisenstein series of weight one}

In this section we begin by considering the simplest case where

$$
g=E_{1}\left(\chi_{g}^{+}, \chi_{g}^{-}\right), \quad h=E_{1}\left(\chi_{h}^{+}, \chi_{h}^{-}\right)
$$

are both Eisenstein series attached to pairs of Dirichlet characters. It is assumed that $\chi_{g}^{+}$is even and $\chi_{g}^{-}$is odd, and likewise for $\chi_{h}^{+}$and $\chi_{h}^{-}$. The nebentype characters of $g$ and $h$ are equal to $\chi_{g}:=\chi_{g}^{+} \chi_{g}^{-}$and $\chi_{h}:=\chi_{h}^{+} \chi_{h}^{-}$respectively.

Fix a prime $p$ not dividing the conductors of $\chi_{g}$ and $\chi_{h}$, and recall we assume the classicality hypothesis on $g$, which in particular implies that $\chi_{g}^{+}(p)=\chi_{g}^{-}(p)$.

The representation $\varrho_{g h}$ decomposes as a direct sum of four one-dimensional characters

$$
\varrho_{g h}=\chi_{g h}^{++} \oplus \chi_{g h}^{--} \oplus \chi_{g h}^{+-} \oplus \chi_{g h}^{-+}:=\chi_{1} \oplus \chi_{2} \oplus \chi_{3} \oplus \chi_{4},
$$

where

$$
\chi_{g h}^{++}:=\chi_{g}^{+} \chi_{h}^{+}, \quad \chi_{g h}^{--}:=\chi_{g}^{-} \chi_{h}^{-}, \quad \chi_{g h}^{+-}:=\chi_{g}^{+} \chi_{h}^{-}, \quad \chi_{g h}^{-+}:=\chi_{g}^{-} \chi_{h}^{+},
$$

Note in particular that the characters $\chi_{g h}^{++}$and $\chi_{g h}^{--}$are even, while the characters $\chi_{g h}^{+-}$and $\chi_{g h}^{-+}$are odd (and in particular, non-trivial).

Given an even character $\chi$ factoring through a finite abelian extension $H_{\chi}$ of $\mathbb{Q}$ and taking values in $L$, denote by $u(\chi) \in L \otimes\left(\mathcal{O}_{H_{\chi}}^{\times}\right)$the fundamental unit in the $\chi$-eigenspace

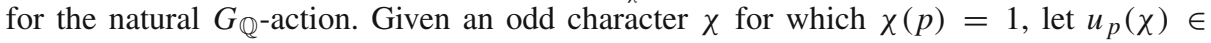
$L \otimes\left(\mathcal{O}_{H_{\chi}}[1 / p]\right)^{\times}$denote the fundamental $p$-unit in the $\chi$-eigenspace for the Galois action.

The form taken on by Conjecture 1.2 depends on the number of even characters among the $\chi_{j}$ which are non-trivial, and hence it is convenient to break up our discussion into three cases:

Case 1 The even characters $\chi_{g h}^{++}$and $\chi_{g h}^{--}$are both non-trivial. In that case, Conjecture 1.2 predicts that $\Xi\left(g_{\alpha}, h\right)=0$ if $\chi_{j}(p)=1$ for some $1 \leq j \leq 4$, and that otherwise

$$
\Xi\left(g_{\alpha}, h\right) \in \Omega_{g h} \cdot M_{1}\left(N_{g h}, \chi_{g}^{-1}\right)\left[g_{\alpha}^{*}\right]_{L} \quad \text { where } \quad \Omega_{g h}=\frac{\log _{p}\left(u\left(\chi_{g h}^{++}\right)\right) \log _{p}\left(u\left(\chi_{g h}^{--}\right)\right)}{\mathfrak{g}\left(\chi_{g h}\right) \log _{p}\left(u_{g_{\alpha}}\right)} .
$$

Case 2 Exactly one of the even characters $\chi_{g h}^{++}$and $\chi_{g h}^{--}$is trivial. Our running assumption that $\chi_{g}^{+}(p)=\chi_{g}^{-}(p)$ implies that $\chi_{g h}^{-+}(p)=1$ if $\chi_{g h}^{++}=1$ and that $\chi_{g h}^{+-}(p)=1$ if $\chi_{g h}^{--}=1$. Conjecture 1.2 predicts that $\Xi\left(g_{\alpha}, h\right)=0$ if $\chi_{j}(p)=1$ for at least two nontrivial characters among the $\chi_{j}$. Assuming that this is not the case, let $\chi_{g h}^{+}$denote the unique non-trivial character in $\left\{\chi_{g h}^{++}, \chi_{g h}^{--}\right\}$and let $\chi_{g h}^{-}$denote the unique character in $\left\{\chi_{g h}^{+-}, \chi_{g h}^{-+}\right\}$ satisfying $\chi_{g h}^{-}(p)=1$. Conjecture 1.2 then predicts that

$$
\Xi\left(g_{\alpha}, h\right) \in \Omega_{g h} \cdot M_{1}\left(N_{g h}, \chi_{g}^{-1}\right)\left[g_{\alpha}^{*}\right]_{L} \quad \text { where } \Omega_{g h}=\frac{\log _{p}\left(u\left(\chi_{g h}^{+}\right)\right) \log _{p}\left(u_{p}\left(\chi_{g h}^{-}\right)\right)}{\mathfrak{g}\left(\chi_{g h}\right) \log _{p}\left(u_{g_{\alpha}}\right)} .
$$

A notable special case arises when $\chi_{g}^{+}=\chi_{h}^{+}=1$ and the odd characters $\chi_{g}^{-}$and $\chi_{h}^{-}$are both quadratic, with $\chi_{g}^{-}(p)=-\chi_{h}^{-}(p)=1$. In that scenario, the $p$-units $u_{p}\left(\chi_{g h}^{-}\right)$and $u_{g_{\alpha}}$ 
that occur in the numerator and denominator of (9) cancel out, and the conjectural formula for the period simplifies to

$$
\Omega_{g h}=\frac{\log _{p}\left(u\left(\chi_{g h}^{--}\right)\right)}{\mathfrak{g}\left(\chi_{g h}\right)} .
$$

One obtains an appealing expression for the $p$-adic logarithm of the fundamental unit of a real quadratic field in terms of $p$-adic iterated integrals. This leads to a (somewhat) new method for solving Pell's equation, albeit one which is hardly an improvement over the classical method of continued fractions!

Case 3 Both of the even characters $\chi_{g h}^{++}$and $\chi_{g h}^{--}$are trivial. This implies that

$$
\chi_{h}^{+}=\left(\chi_{g}^{+}\right)^{-1}, \quad \chi_{h}^{-}=\left(\chi_{g}^{-}\right)^{-1},
$$

so that, after setting $\chi_{g}^{ \pm}:=\chi_{g}^{+} / \chi_{g}^{-}$and $\chi_{g}^{\mp}:=\chi_{g}^{-} / \chi_{g}^{+}$,

$$
\varrho_{g h}=1 \oplus 1 \oplus \chi_{g}^{ \pm} \oplus \chi_{g}^{\mp} .
$$

Both of the odd characters that arise in this decomposition are trivial at $p$. Conjecture 1.2 therefore predicts that $\Xi\left(g_{\alpha}, h\right) \neq 0$ and that

$\Xi\left(g_{\alpha}, h\right) \in \Omega_{g h} \cdot M_{1}\left(N_{g h}, \chi_{g}^{-1}\right)\left[g_{\alpha}^{*}\right]_{L} \quad$ where $\quad \Omega_{g h}=\frac{\log _{p}\left(u_{p}\left(\chi_{g}^{ \pm}\right)\right) \log _{p}\left(u_{p}\left(\chi_{g}^{\mp}\right)\right)}{\log _{p}\left(u_{p}\left(\chi_{g}^{ \pm}\right)\right)+\log _{p}\left(u_{p}\left(\chi_{g}^{\mp}\right)\right)}$.

A notable special case arises when the odd character $\chi_{g}^{ \pm}$is quadratic, and hence equal to $\chi_{g}^{\mp}$. The expression in (11) then simplifies to yield (modulo $L^{\times}$, as always)

$$
\Omega_{g h}=\log _{p}\left(u_{p}\left(\chi_{g}^{ \pm}\right)\right) .
$$

One thus obtains, somewhat like in (10), an appealing expression for the $p$-adic logarithm of the fundamental $p$-unit of an imaginary quadratic field in terms of $p$-adic iterated integrals of Eisenstein series.

The main result of this section is the unconditional proof of certain special cases of Conjecture 1.2 when $g$ and $h$ are both Eisenstein series:

Theorem 3.1 Assume that $g$ and $h$ are Eisenstein series, and that $\chi_{g}^{+}$and $\chi_{g}^{-}$are both quadratic characters of relatively prime conductors. Then Conjecture 1.2 holds, i.e., Eqs. (8), (9) and (11) hold in cases 1,2 and 3 respectively.

Proof Recall that the classicality hypothesis imposed on $g$ implies that

$$
\chi_{g}^{+}(p)=\chi_{g}^{-}(p) .
$$

The pair $\left(\chi_{g}^{+}, \chi_{g}^{-}\right)$corresponds to a genus character $\psi_{g}$ of the imaginary quadratic field $K$ cut out by the odd Dirichlet character $\chi_{g}:=\chi_{g}^{+} \chi_{g}^{-}$, and condition (13) implies that $\chi_{g}(p)=1$, and hence that the prime $\wp=: \mathfrak{p} \overline{\mathfrak{p}}$ splits in $K / \mathbb{Q}$. The weight one Eisenstein series $g=E_{1}\left(\chi_{g}^{+}, \chi_{g}^{-}\right)$is equal to the theta series $\theta_{K}\left(\psi_{g}\right)$ attached to $\psi_{g}$, and the modular form $g$ admits three natural ordinary deformations, arising from the fact that $E_{1}\left(\chi_{g}^{+}, \chi_{g}^{-}\right)=$ $\left.E_{1}\left(\chi_{g}^{-}, \chi_{g}^{+}\right)=\theta_{K}\left(\psi_{g}\right)\right)$ : 
- The Hida families $\underline{E}\left(\chi_{g}^{+}, \chi_{g}^{-}\right)$and $\underline{E}\left(\chi_{g}^{-}, \chi_{g}^{+}\right)$of Eisenstein series, whose weight $k$ specialisations are described by

$$
\underline{E}_{k}\left(\chi_{1}, \chi_{2}\right):=\sum_{n=1}^{\infty} \sigma_{k-1, \chi_{1}, \chi_{2}}^{(p)}(n) q^{n}, \quad \sigma_{k-1, \chi_{1}, \chi_{2}}^{(p)}(n):=\sum_{d \mid n, p \nmid d} \chi_{1}(n / d) \chi_{2}(d) d^{k-1} ;
$$

- The cuspidal family $\underline{\theta}\left(\psi_{g}\right)$ of ordinary theta series whose weight $k$ specialisation is given by

$$
\underline{\theta}_{k}\left(\psi_{g}\right)=\theta\left(\psi_{g} \psi_{p}^{k-1}\right)=\sum_{\mathfrak{a} \subset \mathcal{O}_{K}, \mathfrak{p} \nmid \mathfrak{a}} \psi_{g}(\mathfrak{a}) \psi_{p}^{k-1}(\mathfrak{a}) q^{\mathfrak{a} \overline{\mathfrak{a}}} .
$$

In this equation, the character $\psi_{p}: I(K) \longrightarrow \overline{\mathbb{Q}}_{p} \times$ on the group $I(K)$ of fractional ideals of $K$ is obtained from a canonical Hecke character $\psi_{\infty}: I(K) \longrightarrow \mathbb{C}^{\times}$in the sense of Gross and Rohrlich, sending a principal ideal $(\alpha)$ to $\pm \alpha$ for all $\alpha$ which are relatively prime to the conductor of $\psi_{\infty}$. The character $\psi_{\infty}$ takes its values in a finite extension $L$ of $K$, and $\psi_{p}$ is obtained by composing it with a $p$-adic embedding of $L$ sending $K$ to its completion at $\mathfrak{p}$.

Let $\theta_{3+2 t}\left(\psi_{g}\right)$ denote the weight $3+2 t$ specialisation of the Hida family of theta-series arising from the genus character $\psi_{g}$. The classicality assumption on $\theta_{1}\left(\psi_{g}\right)$ implies that $\underline{\theta}\left(\psi_{g}\right)$ is the unique cuspidal Hida family specialising to $\theta_{1}\left(\psi_{g}\right)$.

Because of this, the $p$-adic iterated integral (1) attached to the pair $\left(\theta_{K}\left(\psi_{g}\right), E_{1}\left(\chi_{h}^{+}, \chi_{h}^{-}\right)\right)$ agrees, up to non-zero algebraic factors, with the $p$-adic limit of the algebraic numbers (depending on $t \geq 0$ )

$$
\Omega_{g h}^{[p]}(t):=\frac{\left\langle\theta_{3+2 t}\left(\psi_{g}\right), d^{t} E_{2}^{[p]}\left(1, \chi_{g h}^{-1}\right) \times E_{1}\left(\chi_{h}^{+}, \chi_{h}^{-}\right)\right\rangle}{\left\langle\theta_{3+2 t}\left(\psi_{g}\right), \theta_{3+2 t}\left(\psi_{g}\right)\right\rangle}
$$

as $t$ tends to -1 in weight space (Note that this ratio does not depend on any choice of normalisation for the Petersson scalar product). Applying Cor. 4.13 of [13] after making the substitutions

$$
(k, \ell, m) \leftarrow(3+2 t, 2,1), \quad f_{k} \leftarrow \theta_{3+2 t}\left(\psi_{g}\right), \quad g \leftarrow E_{2}\left(1, \chi_{g h}^{-1}\right), \quad h \leftarrow E_{1}\left(\chi_{h}^{+}, \chi_{h}^{-}\right),
$$

one obtains

$$
\Omega_{g h}^{[p]}(t)=\frac{\mathcal{E}_{g h}(t)}{\mathcal{E}_{g}(t)} \times \Omega_{g h}(t),
$$

where

$$
\begin{aligned}
\mathcal{E}_{g h}(t):= & \left(1-\psi_{p}(\overline{\mathfrak{p}})^{2+2 t} \chi_{h}^{+}(p) p^{-2-2 t}\right) \times\left(1-\psi_{p}(\overline{\mathfrak{p}})^{2+2 t} \chi_{h}^{-}(p) p^{-2-2 t}\right) \\
& \times\left(1-\psi_{p}(\overline{\mathfrak{p}})^{2+2 t} \bar{\chi}_{h}^{-}(p) p^{-1-2 t}\right) \times\left(1-\psi_{p}(\overline{\mathfrak{p}})^{2+2 t} \bar{\chi}_{h}^{+}(p) p^{-1-2 t}\right), \\
\mathcal{E}_{g}(t):= & \left(1-\psi_{p}(\overline{\mathfrak{p}})^{4+4 t} p^{-3-2 t}\right), \\
\Omega_{g h}(t):= & \frac{\left\langle\theta_{3+2 t}\left(\psi_{g}\right), d^{t} E_{2}\left(1, \chi_{g h}^{-1}\right) \times E_{1}\left(\chi_{h}^{+}, \chi_{h}^{-}\right)\right\rangle}{\left\langle\theta_{3+2 t}\left(\psi_{g}\right), \theta_{3+2 t}\left(\psi_{g}\right)\right\rangle} .
\end{aligned}
$$

It will be convenient to express $\Omega_{g h}^{[p]}(t)$ as a ratio

$$
\Omega_{g h}^{[p]}(t):=\frac{\Omega_{g h}^{\text {num }}(t)}{\Omega_{g}^{\text {den }}(t)}
$$


of two quantities that can (as will be shown) be individually interpolated $p$-adically, namely

$$
\begin{aligned}
\Omega_{g h}^{\text {num }}(t) & :=\frac{\mathcal{E}_{g h}(t) \times \Omega_{K, p}^{4+4 t} \times\left\langle\theta_{3+2 t}\left(\psi_{g}\right), d^{t} E_{2}\left(1, \chi_{g h}^{-1}\right) \times E_{1}\left(\chi_{h}^{+}, \chi_{h}^{-}\right)\right\rangle}{\Omega_{K}^{4+4 t}}, \\
\Omega_{g}^{\operatorname{den}}(t) & :=\frac{\mathcal{E}_{g}(t) \times \Omega_{K, p}^{4+4 t} \times\left\langle\theta_{3+2 t}\left(\psi_{g}\right), \theta_{3+2 t}\left(\psi_{g}\right)\right\rangle}{\Omega_{K}^{4+4 t}}
\end{aligned}
$$

where $\Omega_{K} \in \mathbb{C}^{\times}$is Shimura's canonical period attached to the imaginary quadratic field $K$ and $\Omega_{K, p} \in \mathbb{C}_{p}^{\times}$is the corresponding $p$-adic period which appears in the interpolation formula for the Katz $p$-adic $L$-function. The strategy of proof is to show that both of these functions of $t \in \mathbb{Z}^{\geq 0}$, viewed as taking values in $\mathbb{C}_{p}$, extend to an analytic function on weight space by relating them to the Katz $p$-adic $L$-function, and then to evaluate them at the point $t=-1$ by invoking Katz's $p$-adic analogue of the Kronecker limit formula.

We begin with the expression $\Omega_{g h}^{\text {num }}(t)$. To evaluate it, we invoke formula (18) of [2], after making the substitutions

$$
(k, \ell, m) \leftarrow(3+2 t, 1,2), \quad c \leftarrow 2+t, \quad f \leftarrow \theta_{3+2 t}\left(\psi_{g}\right), \quad g \leftarrow E_{1}\left(\chi_{h}^{+}, \chi_{h}^{-}\right),
$$

to obtain

$$
\left\langle\theta_{3+2 t}\left(\psi_{g}\right), d^{t} E_{2}\left(1, \chi_{g h}^{-1}\right) \times E_{1}\left(\chi_{h}^{+}, \chi_{h}^{-}\right)\right\rangle=C(t) \times L\left(\theta_{3+2 t}\left(\psi_{g}\right) \otimes E_{1}\left(\chi_{h}^{+}, \chi_{h}^{-}\right), 2+t\right),
$$

where

$$
C(t):=\frac{(1+t) !^{2} \times \Omega_{K, p}^{4+4 t}}{(-1)^{t-1} 2^{2+2 t}(2 \pi)^{4+2 t}(-i N)^{-2} \mathfrak{g}\left(\chi_{g h}\right)} .
$$

Formula (19) of loc.cit. implies that

$$
\begin{aligned}
\Omega_{g h}^{\text {num }}(t) & =C(t) \mathcal{E}_{g h}(t) \times \frac{L\left(\theta_{3+2 t}\left(\psi_{g}\right), \chi_{h}^{+}, 2+t\right)}{\Omega_{K}^{2+2 t}} \times \frac{L\left(\theta_{3+2 t}\left(\psi_{g}\right), \chi_{h}^{-}, 2+t\right)}{\Omega_{K}^{2+2 t}} \\
& =C(t) \mathcal{E}_{g h}(t) \times \frac{L\left(\psi_{g} \chi_{h}^{+} \psi_{t}^{-1}, 0\right)}{\Omega_{K}^{2+2 t}} \times \frac{L\left(\psi_{g} \chi_{h}^{-} \psi_{t}^{-1}, 0\right)}{\Omega_{K}^{2+2 t}},
\end{aligned}
$$

where the $L$-functions in the second line are those attached to Hecke characters of $K$, and $\psi_{t}$ is the Hecke character

$$
\psi_{t}:=\psi_{p}^{2+t}\left(\psi_{p}^{\prime}\right)^{-t},
$$

which is of infinity type $(2+t,-t)$, and therefore lies in the range of classical interpolation for the Katz $p$-adic $L$-function precisely when $t \geq 0$.

Let $L_{p}^{\text {Katz }}$ denote the two-variable Katz $p$-adic $L$-function attached to Hecke characters of $K$, and $\Omega_{K, p}$ denote the $p$-adic counterpart of the complex period $\Omega_{K}$ that appears in the interpolation property of $L_{p}^{\text {Katz }}$.

The interpolation formula for the Katz $p$-adic $L$-function, as it is described for instance in $[11, \S 3,(45)]$, shows that

$$
\Omega_{g h}^{\mathrm{num}}(t) \sim_{t} \mathfrak{g}\left(\chi_{g h}\right)^{-1} \times L_{p}^{\mathrm{Katz}}\left(\psi_{g} \bar{\chi}_{h}^{+} \psi_{t}\right) \times L_{p}^{\mathrm{Katz}}\left(\psi_{g} \bar{\chi}_{h}^{-} \psi_{t}\right),
$$

where the symbol $\sim_{t}$ denotes an equality up to an elementary fudge factor which (as a function of the variable $t$ ) extends to an analytic function on weight space and takes $L$-rational, nonzero values at $t=-1$. 
Evaluating this relation at $t=-1$, one finds

$$
\Omega_{g h}^{\text {num }}(-1) \sim \mathfrak{g}\left(\chi_{g h}\right)^{-1} \times L_{p}^{\mathrm{Katz}}\left(\psi_{g} \bar{\chi}_{h}^{+} \mathbf{N}\right) L_{p}^{\mathrm{Katz}}\left(\psi_{g} \bar{\chi}_{h}^{-} \mathbf{N}\right),
$$

where $\mathbf{N}$ denotes the norm character (of infinity type $(1,1)$ ) and we have (in many of the equations above) identified the Dirichlet characters $\chi_{h}^{+}$and $\chi_{h}^{-}$with their restrictions to $G_{K}$, viewed as (finite order) Hecke characters of $K$. Note that the smallest extension $H$ of $K$ cut out by the characters $\psi_{g} \chi_{h}^{+}$and $\psi_{g} \chi_{h}^{-}$is Galois and abelian over $\mathbb{Q}$ as well. If $\psi$ is any non-trivial character of $\operatorname{Gal}(H / K)$, denote by $u_{K}(\psi)$ any element of $L \otimes \mathcal{O}_{H}^{\times}$which generates the $\psi$-eigenspace for the action of $\mathrm{Gal}(H / K)$, and when $\chi$ is trivial, write $u_{K}(1)$ for the fundamental $p$-unit of the imaginary quadratic field $K$. Katz's $p$-adic analogue of the Kronecker limit formula (Cf. for instance [18, §10.4, 10.5], [17, p. 90], [9, Ch. II,§5.2]) shows that

$$
L_{p}^{\mathrm{Katz}}\left(\psi_{g} \bar{\chi}_{h}^{+} \mathbf{N}\right)= \begin{cases}0 & \text { if } \psi_{g} \chi_{h}^{+} \neq 1, \psi_{g} \chi_{h}^{+}(p)=1 ; \\ \log _{p}\left(u_{K}\left(\psi_{g} \chi_{h}^{+}\right)\right) & \text {otherwise. }\end{cases}
$$

Because the character $\psi_{g} \chi_{h}^{+}$arises from the restriction of a character of $\mathbb{Q}$ and $\psi_{g}$ is the genus character attached to the pair $\left(\chi_{g}^{+}, \chi_{g}^{-}\right)$of quadratic Dirichlet characters, it can be seen that

$$
u_{K}\left(\psi_{g} \chi_{h}^{+}\right)= \begin{cases}u\left(\chi_{g h}^{++}\right) & \text {if } \psi_{g} \chi_{h}^{+} \neq 1 \\ u_{p}\left(\chi_{g h}^{-+}\right) & \text {if } \psi_{g} \chi_{h}^{+}=1\end{cases}
$$

Therefore, it follows that

$$
L_{p}^{\mathrm{Katz}}\left(\psi_{g} \bar{\chi}_{h}^{+} \mathbf{N}\right) \sim \begin{cases}0 & \text { if } \chi_{g h}^{++} \neq 1, \text { and } \chi_{h}^{+}(p)=\chi_{g}^{+}(p)=\chi_{g}^{-}(p) ; \\ \log _{p}\left(u\left(\chi_{g h}^{++}\right)\right) & \text {if } \chi_{g h}^{++} \neq 1, \text { and } \chi_{h}^{+}(p) \neq \chi_{g}^{+}(p) \\ \log _{p}\left(u_{p}\left(\chi_{g h}^{-+}\right)\right) & \text {if } \chi_{g h}^{++}=1 .\end{cases}
$$

Similar considerations applied to the character $\psi_{g} \chi_{h}^{-}$show that

$$
L_{p}^{\mathrm{Katz}}\left(\psi_{g} \bar{\chi}_{h}^{-} \mathbf{N}\right) \sim \begin{cases}0 & \text { if } \chi_{g h}^{--} \neq 1, \text { and } \chi_{h}^{-}(p)=\chi_{g}^{+}(p)=\chi_{g}^{-}(p) ; \\ \log _{p}\left(u\left(\chi_{g h}^{--}\right)\right) & \text {if } \chi_{g h}^{--} \neq 1, \text { and } \chi_{h}^{-}(p) \neq \chi_{g}^{-}(p) \\ \log _{p}\left(u_{p}\left(\chi_{g h}^{+-}\right)\right) & \text {if } \chi_{g h}^{--}=1 .\end{cases}
$$

It follows that

$$
\Omega_{g h}^{\text {num }}(-1) \sim \begin{cases}\frac{\log _{p}\left(\chi_{g h}^{++}\right) \log _{p}\left(\chi_{g h}^{--}\right)}{\mathfrak{g}\left(\chi_{g h}\right)} & \text { in case 1; } \\ \frac{\log _{p}\left(u\left(\chi_{g h}^{+}\right) \log _{p}\left(u_{p}\left(\chi_{g h}^{-}\right)\right)\right.}{\mathfrak{g}\left(\chi_{g h}\right)}, & \text { in case 2; } \\ \log _{p}\left(u_{p}\left(\chi_{g}^{ \pm}\right)\right) \log _{p}\left(u_{p}\left(\chi_{g}^{\mp}\right)\right) & \text { in case 3. }\end{cases}
$$

In addition, it follows from [11, Lemma 3.8], where $t=k-2$, that $\Omega_{g}^{\text {den }}(t)$ likewise extends to a $p$-adic analytic function of $t$, and

$$
\Omega_{g}^{\mathrm{den}}(-1) \sim \log _{p}\left(u_{p}\left(\chi_{g}\right)\right)
$$

by [11, Theorem 3.9 (54)]. 
The theorem follows directly from Eqs. (17) and (18) in light of the fact that

$$
\Omega_{g h} \sim \frac{\Omega_{g h}^{\text {num }}(-1)}{\Omega_{g}^{\operatorname{den}}(-1)} .
$$

Remark 3.2 The analogue of Theorem 3.1 in the setting explored in [11, §7], where the weight two Eisenstein series $E_{2}\left(1, \chi_{g h}^{-1}\right)$ is replaced by a cusp form $f$ attached to an elliptic curve $E$ over $\mathbb{Q}$, poses significant difficulties and remains unproved. This analogue would involve points on $E$ defined over the cyclotomic fields cut out by the characters $\chi_{g h}^{++}, \chi_{g h}^{+-}$, $\chi_{g h}^{-+}$, and $\chi_{g h}^{--}$, and no appropriate variant of the Heegner construction leading to such points is available, at least when the characters involved are non quadratic. Such a variant would occupy the same position vis-à-vis Heegner points as circular units vis-à-vis elliptic units.

\section{Numerical examples}

Example 3.3 The following experiments illustrate Theorem 3.1 in the special case where $g=E_{1}\left(1, \chi_{g}^{-}\right)$and $h=E_{1}\left(1, \chi_{h}^{-}\right)$, where $\chi_{g}^{-}$and $\chi_{h}^{-}$are odd quadratic Dirichlet characters satisfying $\left(\chi_{g}^{-}(p), \chi_{h}^{-}(p)\right)=(1,-1)$. This corresponds to the special case of Conjecture 1.2 described in Eq. (10) of Case 2 treated above, involving the $p$-adic logarithm of the fundamental unit of the real quadratic field cut out by the even character $\chi_{g h}^{--}$.

3.3a. For this experiment, let $\chi_{g}^{-}$and $\chi_{h}^{-}$be the odd quadratic Dirichlet characters of conductors 3 and 11 respectively, and choose $p:=7$. The 7-stabilised eigenform $g_{\alpha}:=E_{1}^{(7)}\left(1, \chi_{g}^{-}\right)$ (of level $3 \cdot 7$ ) has two distinct 11-stabilisations, denoted $g_{1}$ and $g_{-1}$, of level $3 \cdot 7 \cdot 11$, with $U_{11}$-eigenvalues 1 and -1 , respectively. Denote by $\gamma_{1}$ and $\gamma_{-1}$ the linear functionals on $S_{1}(3 \cdot 7 \cdot 11)\left[g_{\alpha}\right]=\mathbb{C}_{p} g_{1} \oplus \mathbb{C}_{p} g_{-1}$ sending $\left(g_{1}, g_{-1}\right)$ to $(1,0)$ and $(0,1)$ respectively. A computer calculation using the algorithms based on [19] shows that

$$
\begin{aligned}
& \int_{\gamma_{1}} E_{2}\left(1, \chi_{g h}^{--}\right) \cdot E_{1}\left(1, \chi_{h}^{-}\right)=-9955228630224805563482516 \quad\left(\bmod 7^{30}\right) \\
& \int_{\gamma_{-1}} E_{2}\left(1, \chi_{g h}^{--}\right) \cdot E_{1}\left(1, \chi_{h}^{-}\right)=0 \quad\left(\bmod 7^{30}\right) .
\end{aligned}
$$

A careful examination of the non-vanishing 7-adic iterated integral above suggests that

$$
\int_{\gamma_{1}} E_{2}\left(1, \chi_{g h}^{--}\right) \cdot E_{1}\left(1, \chi_{h}^{-}\right)=-\frac{2 \cdot \sqrt{33} \cdot \log _{7}\left(u\left(\chi_{g h}^{--}\right)\right)}{7}
$$

to 30 digits of 7-adic precision, where $u\left(\chi_{g h}^{--}\right)$is the fundamental unit in the real quadratic field cut out by the even character $\chi_{g h}^{--}$, satisfying the polynomial $x^{2}-46 x+1$.

3.3b. We place ourselves in exactly the same settting as in $3.3 \mathrm{a}$, but taking this time $\chi_{h}^{-}$to be the odd quadratic character of conductor 23 rather than 11. Adopting the same notations as for the previous example, we find:

$$
\begin{aligned}
& \int_{\gamma_{1}} E_{2}\left(1, \chi_{g h}^{--}\right) \cdot E_{1}\left(1, \chi_{h}^{-}\right)=10649075716435865817575346 \quad\left(\bmod 7^{30}\right) \\
& \int_{\gamma_{-1}} E_{2}\left(1, \chi_{g h}^{--}\right) \cdot E_{1}\left(1, \chi_{h}^{-}\right)=0 \quad\left(\bmod 7^{30}\right)
\end{aligned}
$$


and

$$
\int_{\gamma_{1}} E_{2}\left(1, \chi_{g h}^{--}\right) \cdot E_{1}\left(1, \chi_{h}^{-}\right)=-\frac{3 \cdot \sqrt{69} \cdot \log _{7}\left(u\left(\chi_{g h}^{--}\right)\right)}{7}
$$

to 30 digits of 7 -adic precision.

3.3c. In the third experiment of this kind, we take $\chi_{g}^{-}$to have conductor 7 and $\chi_{h}^{-}$to have conductor 3 , with $p:=11$. Then

$$
\begin{aligned}
& \int_{\gamma_{1}} E_{2}\left(1, \chi_{g h}^{--}\right) \cdot E_{1}\left(1, \chi_{h}^{-}\right)=-11038147038719013039751993 \quad\left(\bmod 11^{25}\right) \\
& \int_{\gamma_{-1}} E_{2}\left(1, \chi_{g h}^{--}\right) \cdot E_{1}\left(1, \chi_{h}^{-}\right)=0 \quad\left(\bmod 11^{25}\right)
\end{aligned}
$$

and we find again that

$$
\int_{\gamma_{1}} E_{2}\left(1, \chi_{g h}^{--}\right) \cdot E_{1}\left(1, \chi_{h}^{-}\right)=-\frac{\sqrt{21} \cdot \log _{11}\left(u\left(\chi_{g h}^{--}\right)\right)}{11}
$$

to 25 digits of 11 -adic precision.

Example 3.4 Let $\chi_{3}$ and $\chi_{4}$ be the odd quadratic characters of conductors 3 and 4 , respectively, and let $\chi_{5}$ be the even quadratic character of conductor 5 . Let $g:=E_{1}\left(1, \chi_{3}\right)$ and $h:=E_{1}\left(\chi_{5}, \chi_{4}\right)$. We have found experimentally that the 7 -adic iterated integrals attached to $g$ and $h$, for all the stabilisations of $g$ in level $3 \cdot 7 \cdot 4 \cdot 5$, are all zero, except for

$$
\int_{\gamma} E_{2}\left(1, \chi_{3} \cdot \chi_{4} \cdot \chi_{5}\right) \cdot E_{1}\left(\chi_{5}, \chi_{4}\right)=-1510481263266793866295817 \quad\left(\bmod 7^{30}\right) \text {, }
$$

where $\gamma$ is the unique element of the dual space of $S_{1}\left(7 \cdot 60, \chi_{3}\right)\left[g_{\alpha}\right]$ sending the stabilisation of $g$ with $U_{2}$ and $U_{5}$ eigenvalues 1 and -1 , respectively to 1 , and all other stabilisations to 0 . Experimentally, one finds

$$
\int_{\gamma} E_{2}\left(1, \chi_{3} \cdot \chi_{4} \cdot \chi_{5}\right) \cdot E_{1}\left(\chi_{5}, \chi_{4}\right)=\frac{2^{4} \cdot \sqrt{60} \cdot \log _{7}\left(u\left(\chi_{5}\right)\right) \cdot \log _{7}\left(u\left(\chi_{12}\right)\right)}{3 \cdot 7 \cdot \log _{7}\left(u_{7}\left(\chi_{3}\right)\right)}
$$

to 30-digits of 7-adic precision, in perfect agreement with the most "generic" instance of Conjecture 1.2 treated in Case 1 above.

\section{Elliptic units and theta series of imaginary quadratic fields}

\subsection{Theoretical evidence}

Let $K$ be an imaginary quadratic field of discriminant $-D_{K}$ and let $\chi_{K}$ denote the quadratic Dirichlet character associated to it. The aim of this chapter is to study Conjecture 1.2 in the case where both $g$ and $h$ are theta series associated to $K$.

Fix two arbitrary finite order characters $\psi_{g}, \psi_{h}$ of $K$ of conductors $\mathfrak{c}_{g}, \mathfrak{c}_{h}$. Let $\chi_{g}, \chi_{h}$ denote the central characters of $\psi_{g}$ and $\psi_{h}$. Let

$$
g:=\theta_{\psi_{g}} \in M_{1}\left(N_{g}, \chi_{K} \chi_{g}\right), \quad h:=\theta_{\psi_{h}} \in M_{1}\left(N_{h}, \chi_{K} \chi_{h}\right)
$$

be the theta series associated to them. Here $N_{g}=D_{K} \cdot N_{K / \mathbb{Q}}\left(\mathfrak{c}_{g}\right)$ and $N_{h}=D_{K} \cdot N_{K / \mathbb{Q}}\left(\mathfrak{c}_{h}\right)$.

Define $\psi_{1}=\psi_{g} \psi_{h}$ and $\psi_{2}=\psi_{g} \psi_{h}^{\prime}$ where, for any character $\psi$ of $G_{K}$, we denote $\psi^{\prime}$ the character given by the rule $\psi^{\prime}(\sigma)=\psi\left(\sigma_{0} \sigma \sigma_{0}^{-1}\right)$ for any choice of $\sigma_{0} \in G_{\mathbb{Q}}-G_{K}$. Unlike 
in $[11, \S 3]$, here we are not imposing the restriction $\chi_{g}=\chi_{h}^{-1}$, and thus $\psi_{1}$ and $\psi_{2}$ are not necessarily ring class characters.

Let $V_{g}=\operatorname{Ind}_{K}^{\mathbb{Q}}\left(\psi_{g}\right)$ and $V_{h}=\operatorname{Ind}_{K}^{\mathbb{Q}}\left(\psi_{h}\right)$ denote the two-dimensional induced representations of $\psi_{g}$ and $\psi_{h}$ from $G_{K}$ to $G_{\mathbb{Q}}$, with coefficients in a finite extension $L$ of $\mathbb{Q}$ which we may take to contain the fourier coefficients of $g$ and $h$ and the Gauss sum $\mathfrak{g}\left(\chi_{g h}\right)$. By Lemma 2.1, there is a decomposition of Artin representations

$$
V_{g h}=V_{\psi_{g}} \otimes V_{\psi_{h}} \simeq V_{\psi_{1}} \oplus V_{\psi_{2}}
$$

where for $i=1,2, V_{\psi_{i}}$ is irreducible if and only if $\psi_{i} \neq \psi_{i}^{\prime}$. If $\psi_{i}=\psi_{i}^{\prime}$, let $\chi_{i}$ denote the central character of $\psi_{i}$. Then $V_{\psi_{i}} \simeq \chi_{i} \oplus \chi_{K} \chi_{i}$ decomposes as the sum of an odd and even Dirichlet character. The odd one can never be trivial, while the even one is trivial precisely when $\psi_{i}=1$.

Let $N=\operatorname{gcd}\left(N_{g}, N_{h}\right)$ and let $p \nmid N$ be a prime that splits in $K$. By Lemma 1.1, it follows that

$$
d_{g h}^{(p)}=2 \text { if and only if } \psi_{g} \neq \psi_{h}^{-1},\left(\psi_{h}^{\prime}\right)^{-1},
$$

which we assume for the remainder of this chapter. In that case we in fact have $d_{g h}=d_{g h}^{(p)}=2$ and, more precisely,

$$
\operatorname{dim}_{L}\left(V_{\psi_{i}}, U_{g h}\right)=1 \quad \text { for } i=1,2 .
$$

Write $U_{g h}^{\psi_{i}}$ for the image of $V_{\psi_{i}}$ in $U_{g h}$. The theory of elliptic units allows us to pin down distinguished choices of elements in $U_{g h}^{\psi_{i}}$ as follows.

Let $H_{g} / K$ and $H_{h} / K$ denote the abelian extensions cut out by the characters $\psi_{g}$ and $\psi_{h}$, respectively, and let $H_{g h}=H_{g} H_{h}$ denote their composition. Since $p$ splits in $K$, the frobenius element $\sigma_{p}$ belongs to $\mathrm{Gal}\left(H_{g h} / K\right)$, and the choice of a prime $\wp$ of $K$ above $p$ is sufficient to determine $\sigma_{p}$ as an element of this group. The choices of $\alpha_{g}, \beta_{g}, \alpha_{h}$ and $\beta_{h}$ can then be made so that

$$
\alpha_{g}=\psi_{g}(\wp), \quad \beta_{g}=\psi_{g}(\bar{\wp}), \quad \alpha_{h}=\psi_{h}(\wp), \quad \beta_{h}=\psi_{h}(\bar{\wp}) .
$$

Let $K_{\mathfrak{f}}$ denote the smallest ray class field containing $H_{g h}$ and let $u \in K_{\mathfrak{f}}^{\times}$be an elliptic unit constructed as in $[9, \S 2.6]$. For any character $\psi$ of $G=\operatorname{Gal}\left(K_{\mathfrak{f}} / K\right)$, define

$$
u_{\psi}=\sum_{\sigma \in G} \psi\left(\sigma^{-1}\right) u^{\sigma} .
$$

If $\psi \neq 1$, the element $\sum \psi\left(\sigma^{-1}\right) \cdot \sigma$ belongs to the augmentation ideal of $L[G]$ and [9, $\S 2.6]$ shows that $u_{\psi}$ lies in $L \otimes \mathcal{O}_{K_{\mathfrak{f}}}^{\times}$. Since, by (20), the characters $\psi_{i}, i=1,2$, are not trivial, the four units $u_{\psi_{1}}, u_{\psi_{1}^{\prime}}, u_{\psi_{2}}, u_{\psi_{2}^{\prime}}$ lie in $U_{g h}$. In fact, by construction $u_{\psi_{1}}$ belongs to $U_{g h}^{\psi_{1}}$ and $\sigma_{p}$ acts on it with eigenvalue $\alpha_{g} \alpha_{h}$. Analogous properties hold for $u_{\psi_{1}^{\prime}} \in U_{g h}^{\psi_{1}}$ and $u_{\psi_{2}}, u_{\psi_{2}^{\prime}} \in U_{g h}^{\psi_{2}}$.

Both when $g$ is cuspidal or Eisenstein, the matrix in (4) is

$$
R_{g_{\alpha}}\left(\varrho_{g h}\right)=\left(\begin{array}{ll}
\log _{p}\left(u_{\psi_{1}}\right) & 0 \\
0 & \log _{p}\left(u_{\psi_{2}}\right)
\end{array}\right) .
$$

In addition to the above units, let us introduce also a canonical choice for the unit $u_{g_{\alpha}}$ entering in Conjecture 1.2. 
If $g$ is cuspidal, let $\psi_{\mathrm{ad}}:=\psi_{g} / \psi_{g}^{\prime}$ denote the character associated to the adjoint of $g$ and define $u_{g_{\alpha}}=u_{\psi_{\mathrm{ad}}}$. Since $\psi_{g} \neq \psi_{g}^{\prime}$, it follows as above that $u_{g_{\alpha}}$ belongs to $L \otimes \mathcal{O}_{H_{g}}^{\times}$and that $\sigma_{p}$ acts on it with eigenvalue $\alpha_{g} / \beta_{g}$.

If $g$ is Eisenstein, i.e. $\psi_{g}=\psi_{g}^{\prime}$, define

$$
u_{g_{\alpha}}:=u_{\wp} \in L \otimes \mathcal{O}_{H_{g}}[1 / p]^{\times}
$$

to be any element such that $\wp^{h_{K}}=\left(u_{\wp}\right)$, where $h_{K}$ denotes the class number of $K$.

Conjecture 1.2 then predicts that

$$
\Xi\left(g_{\alpha}, h\right) \in \Omega_{g h} \cdot M_{1}\left(N_{g h}, \chi_{g}^{-1}\right)\left[g_{\alpha}^{*}\right]_{L} \quad \text { where } \Omega_{g h}=\frac{\log _{p}\left(u_{\psi_{1}}\right) \log _{p}\left(u_{\psi_{2}}\right)}{\log _{p}\left(u_{g_{\alpha}}\right)} .
$$

The main result of this section is:

Theorem 4.1 Conjecture 1.2 holds in the setting described above, i.e., (23) holds true.

The remainder of this section is devoted to the proof of Theorem 4.1. Fix a Hecke character $\lambda$ of infinity type $(1,0)$ and conductor $\wp$. For every integer $k \geq 1$ define $\psi_{g, k-1}^{(p)}=\psi_{g}\langle\lambda\rangle^{k-1}$ and let $\psi_{g, k-1}$ be the Hecke character of infinity type $(k-1,0)$ given by

$$
\psi_{g, k-1}(\mathfrak{q})= \begin{cases}\psi_{g, k-1}^{(p)}(\mathfrak{q}) & \text { if } \mathfrak{q} \neq \wp \\ \chi_{g}(p) p^{k-1} / \psi_{g, k-1}^{(p)}(\bar{\wp}) & \text { if } \mathfrak{q}=\wp .\end{cases}
$$

Let $g_{k}=\theta\left(\psi_{g, k-1}\right)$ denote the theta series associated to $\psi_{g, k-1}$, an eigenform of weight $k$. These modular forms vary $p$-adically, in the sense that there is a Hida family $\mathbf{g}$ of tame level $N_{g}$ and character $\chi_{g}$ over an open subset $\mathcal{U}_{g} \subset 1+p \mathbb{Z}_{p}$ of weight space such that its classical specializations are the ordinary $p$-stabilizations of $g_{k}$. Note that $\psi_{g, 1}=\psi_{g}$ and thus $g=g_{1}$; the specialization of $\mathbf{g}$ in weight one is $g_{\alpha}$.

For the remainder of this section, we replace the Hida family $\mathbf{g}$ with a $\Lambda$-adic modular form $\breve{\mathbf{g}}(q)=\sum_{d \mid \frac{N}{N g}} \lambda_{d} \mathbf{g}\left(q^{d}\right)$ of tame level $N$ satisfying that all its classical specializations are not only eigenvectors for the Hecke operators $T_{\ell}$ for $\ell \nmid N p$ but also for the operators $U_{q}$ for all $q \mid N p$. Likewise, replace $h$ with an oldform $\breve{h} \in M_{1}\left(N, \chi_{h}\right)$ that is an eigenvector for all good and bad Hecke operators.

Together with (24), define also the Hecke characters

$$
\Psi_{g h}(k)=\left(\psi_{g, k-1}^{\prime}\right)^{-1} \cdot \psi_{h}^{-1} \cdot \mathbf{N}^{\frac{k+1}{2}} \text { and } \Psi_{g h^{\prime}}(k)=\left(\psi_{g, k-1}^{\prime}\right)^{-1} \cdot\left(\psi_{h}^{\prime}\right)^{-1} \cdot \mathbf{N}^{\frac{k+1}{2}},
$$

where $\mathbf{N}$ is the Hecke character induced by the norm from $K$ to $\mathbb{Q}$.

It follows immediately from the definitions that, for all $k \geq 1$, the Rankin-Selberg $L$ function $L\left(\breve{g}_{k}, \breve{h}, s\right)$ factors as the product of the two $L$-series associated to the (inverse of the) Hecke characters appearing in (25), that is to say:

$$
L\left(\breve{g}_{k}, \breve{h}, \frac{k+1}{2}\right)=\mathfrak{f}_{0}(k) \cdot L\left(\Psi_{g h}^{-1}(k), 0\right) \cdot L\left(\Psi_{g h^{\prime}}^{-1}(k), 0\right) .
$$

Here $\mathfrak{f}_{0}(k)$ is a fudge factor which is a rational function on $q^{k}$ as $q$ ranges over the divisors of $N$, with coefficients in $L$. Moreover, $\mathfrak{f}_{0}(k) \in L^{\times}$for all $k \in \mathbb{Z}_{\geq 1}$. In the terminology coined in [11, Definition 4.3], functions satisfying these properties are called $L$-admissible, and we identify them with their unique analytic extension to $\mathcal{U}_{g}$. 
The factorisation formula (26) admits a $p$-adic analogue, of which Theorem 4.1 is a consequence, as we show later. Since the reader may find it of independent interest, we state it below as a separate result. For every $k \geq 1$, define the Hecke character

$$
\Psi_{g}(k):=\left(\psi_{g, k-1}^{\prime}\right)^{-2} \chi_{g} \mathbf{N}^{k} .
$$

Let $\mathscr{L}_{p}(\breve{\mathbf{g}}, \breve{h})$ denote Hida's $p$-adic Rankin $L$-function on $\mathcal{U}_{g}$ associated to the pair $(\breve{\mathbf{g}}, \breve{h})$. We refer e.g. to [3] for its precise definition, and we shall use the normalizations adopted therein.

As in the previous chapter, let also $L_{p}^{\mathrm{Katz}}$ denote Katz's $p$-adic $L$-function, defined and normalized as in [11].

Theorem 4.2 There is an L-admissible function $\mathfrak{f}$ such that the following factorisation of analytic functions on $\mathcal{U}_{\mathrm{g}}$ is valid:

$$
\mathscr{L}_{p}(\breve{\mathbf{g}}, \breve{h})(k) \cdot L_{p}^{\mathrm{Katz}}\left(\Psi_{g}(k)\right)=\mathfrak{f}(k) \cdot L_{p}^{\mathrm{Katz}}\left(\Psi_{g h}(k)\right) \cdot L_{p}^{\mathrm{Katz}}\left(\Psi_{g h^{\prime}}(k)\right) .
$$

Proof It suffices to show that (27) holds true for all $k \in U_{g}:=\mathcal{U}_{g} \cap \mathbb{Z}_{\geq 2}$, as this is a dense subset of $\mathcal{U}_{g}$ for the $p$-adic topology. Since points in $U_{g}$ belong to the region of interpolation of the four $p$-adic $L$-functions appearing in (27), their values are non-zero multiples of critical classical $L$-values. Namely, there are explicit functions $\Omega_{i}(k), i=1, \ldots, 4$, such that

$$
\begin{aligned}
& \mathscr{L}_{p}(\breve{\mathbf{g}}, \breve{h})(k)=\Omega_{1}(k) \cdot L\left(\breve{g}_{k}, \breve{h}, \frac{k+1}{2}\right), \quad L_{p}^{\mathrm{Katz}}\left(\Psi_{g}(k)\right)=\Omega_{2}(k) \cdot L\left(\Psi_{g}^{-1}(k), 0\right), \\
& L_{p}^{\mathrm{Katz}}\left(\Psi_{g h}(k)\right)=\Omega_{3}(k) \cdot L\left(\Psi_{g h}^{-1}(k), 0\right), \quad L_{p}^{\mathrm{Katz}}\left(\Psi_{g h^{\prime}}(k)\right)=\Omega_{4}(k) \cdot L\left(\Psi_{g h^{\prime}}^{-1}(k), 0\right) .
\end{aligned}
$$

Functions $\Omega_{i}(k)$ involve powers of suitable complex and $p$-adic periods and products of Euler-like factors at primes dividing $p N$. The reader may find completely explicit formulas for them in $[3, \S 2]$ and $[11, \S 3.2]$, respectively. In particular, it is apparent from these expressions that each $\Omega_{i}(k)$ individually does not interpolate $p$-adically to a continuous function on $\mathcal{U}_{g}$, thus a fortiori they are not $L$-admissible at all. However, [11, Lemma3.7] together with a rather tedious but elementary computation show that the ratio

$$
\mathfrak{f}(k):=\frac{\Omega_{1}(k) \Omega_{2}(k)}{\Omega_{3}(k) \Omega_{4}(k)} \cdot L\left(\Psi_{g}^{-1}(k), 0\right)
$$

does vary $p$-adically continuously, and gives rise to an $L$-admissible function. We omit the details, since the proof of this fact proceeds along similar lines as in [11, Theorem 3.8], although the calculations are much simpler than in loc. cit. because here we avoid the subtleties arising in [11] from the choice of test vectors for Garrett's triple-product $L$-function.

The theorem now follows from (27).

In order to prove Theorem 4.1, we proceed by relating the values at $k=1$ of the functions appearing in (27) to a $p$-adic iterated integral and the $p$-adic logarithm of suitable units. Note that

$$
\begin{aligned}
\Psi_{g h}(1)= & \left(\psi_{g}^{\prime}\right)^{-1} \cdot \psi_{h}^{-1} \cdot \mathbf{N}, \quad \Psi_{g h^{\prime}}(1)=\left(\psi_{g}^{\prime}\right)^{-1} \cdot\left(\psi_{h}^{\prime}\right)^{-1} \cdot \mathbf{N} \\
& \text { and } \Psi_{g}(1)=\left(\psi_{g}^{\prime}\right)^{-2} \chi_{g} \mathbf{N}=\frac{\psi_{g}}{\psi_{g}^{\prime}} \mathbf{N},
\end{aligned}
$$

Since for any finite order character $\psi$ of $G_{K}$, Katz's $p$-adic $L$-function satisfies the functional equation

$$
L_{p}^{\mathrm{Katz}}(\psi)=L_{p}^{\mathrm{Katz}}\left(\left(\psi^{\prime}\right)^{-1} \mathbf{N}_{K}\right),
$$


Theorem 4.2 specializes to the following formula at $k=1$ :

$$
\mathscr{L}_{p}(\breve{\mathbf{g}}, \breve{h})(k) \cdot L_{p}^{\mathrm{Katz}}\left(\frac{\psi_{g}}{\psi_{g}^{\prime}}\right)=\mathfrak{f}(1) \cdot L_{p}^{\mathrm{Katz}}\left(\psi_{g} \psi_{h}^{\prime}\right) \cdot L_{p}^{\mathrm{Katz}}\left(\psi_{g} \psi_{h}\right) .
$$

Lemma 4.3 Let $g_{\alpha}^{*}=g_{\alpha} \otimes \chi_{g}^{-1}$ denote the twist of $g_{\alpha}$ under the inverse of its nebentypus. There exists a linear functional

$$
\breve{\gamma}_{\alpha}: M_{1}\left(N_{g h} p, \chi_{g}^{-1}\right)_{L}\left[g_{\alpha}^{*}\right] \longrightarrow L
$$

such that

$$
\mathscr{L}_{p}(\breve{\mathbf{g}}, \breve{h})(1)=\int_{\breve{\gamma_{\alpha}}} f \cdot \breve{h} .
$$

Proof Since the $p$-adic $L$-function $\mathscr{L}_{p}(\breve{\mathbf{g}}, \breve{h})$ of $[3, \S 2.2 .2]$ is constructed using the method of [13], the proof of this statement follows verbatim as in [11, Proposition2.5].

In addition to the above lemma, recall Katz's $p$-adic analogue of Kronecker's limit formula, which asserts that for any finite order character $\psi$ of $G_{K}$, the following equality holds up to scalars in $L^{\times}$:

$$
L_{p}^{\mathrm{Katz}}(\psi)= \begin{cases}\log _{p}\left(u_{\psi}\right) & \text { if } \psi \neq 1 \\ \log _{p}\left(u_{\wp}\right) & \text { if } \psi=1,\end{cases}
$$

where $u_{\psi}$ and $u_{\wp}$ are as in (21) and (22) respectively.

Since $\mathfrak{f}$ is $L$-admissible, $\mathfrak{f}(1)$ is a non-zero scalar in $L$. Theorem 4.1 follows, as the combination of (28), Lemma 4.3 and (30) show that

$$
\breve{\gamma}_{\alpha}\left(\Xi\left(g_{\alpha}, h\right)\right)=\lambda \cdot \frac{\log _{p}\left(u_{\psi_{1}}\right) \log _{p}\left(u_{\psi_{2}}\right)}{\log _{p}\left(u_{g_{\alpha}}\right)}
$$

for some $\lambda \in L^{\times}$.

Example 4.4 We revisit the setting treated in [11, Example 4.3]. Let $\chi_{5}$ and $\chi_{31}$ denote the (even and odd, respectively) quadratic Dirichlet characters of conductor 5 and 31, and let $\chi:=\chi_{5} \cdot \chi_{31}$ be the quadratic character attached to the quadratic field $K:=\mathbb{Q}(\sqrt{-155})$. The class group of $K$ is cyclic of order 4 , and the two-dimensional representation induced from a quartic character $\psi$ of this class group is attached to the weight one cuspidal eigenform

$$
g=\theta_{\psi}=q+q^{4}-q^{5}-q^{9}+q^{16}-2 q^{19}-\cdots \in S_{1}(155, \chi) .
$$

The Galois representation $\varrho_{g}$ attached to $g$ has image the dihedral group $D_{4}$ of order 8 , and projective image isomorphic to the Klein 4-group. It is also induced from quadratic characters of $\mathbb{Q}(\sqrt{5})$ and $\mathbb{Q}(\sqrt{-31})$ (ramified at a prime above 31 and 5 , respectively). In particular, the classicality hypothesis for $g$ is satisfied for the prime $p$ precisely when $\chi_{5}(p)=-1$. Set $h:=g=g^{*}$ and let $f:=E_{2}^{(p)}(1,1)$ be the Eisenstein series of weight 2 and level $p$ attached to the trivial character.

Although $g=h=\theta_{\psi}$ is cuspidal, the tensor product $\varrho_{g h}$ decomposes as a direct sum

$$
\varrho_{g h}=1 \oplus \chi_{5} \oplus \chi_{31} \oplus \chi_{155},
$$

while

$$
\operatorname{Ad}\left(\varrho_{g}\right)=\chi_{5} \oplus \chi_{31} \oplus \chi_{155}
$$


The period $\Omega_{g h}$ of Conjecture 1.2 involves the $p$-adic logarithms of the fundamental unit $u\left(\chi_{5}\right)$ of the quadratic field $\mathbb{Q}(\sqrt{5})$ in both its numerator and denominator. This quantity cancels out in the conjecture, which then predicts that the iterated integrals attached to $(f, g, g)$ should agree with the logarithm of the fundamental $p$-unit of the imaginary quadratic field $K=\mathbb{Q}(\sqrt{-155})$, if $\chi_{155}(p)=1$ (resp. of $\mathbb{Q}(\sqrt{-31})$, if $\chi_{31}(p)=1$ ). The running assumption that $\chi_{5}(p)=-1$ ensures that one is always in precisely one of these two scenarios.

The prime $p:=7$ is inert in $K / \mathbb{Q}$, and the frobenius eigenvalues $\alpha_{g}$ and $\beta_{g}$ are equal to 1 and -1 respectively. Letting $\gamma_{1}$ and $\gamma_{-1}$ denote the associated elements in the dual space of $S_{1}(155, \chi)$, we calculate:

$$
\int_{\gamma_{ \pm 1}} E_{2}^{(7)}(1,1) \cdot \theta_{\psi}=4365724287399866821223816\left(\bmod 7^{30}\right) \text {, }
$$

and check to 30 digits of 7 -adic precision that

$$
\int_{\gamma_{ \pm 1}} E_{2}^{(7)}(1,1) \cdot \theta_{\psi}=-\frac{\log _{7}\left(u_{7}\left(\chi_{31}\right)\right)}{7} .
$$

The prime $p:=13$ splits in $K / \mathbb{Q}$, and the associated frobenius eigenvalues are $i$ and $-i$. A numerical calculation reveals that

$$
\int_{\gamma_{ \pm i}} E_{2}^{(13)}(1,1) \cdot \theta_{\psi}=11525893437665284\left(\bmod 13^{15}\right) \text {. }
$$

One observes, to 15 digits of 13 -adic precision, that

$$
\int_{\gamma_{ \pm i}} E_{2}^{(13)}(1,1) \cdot \theta_{\psi}=-\frac{3 \cdot \log _{13}\left(u_{13}\left(\chi_{155}\right)\right)}{13}
$$

as predicted by Conjecture 1.2.

\section{Gross-Stark units and theta series of real quadratic fields}

The setting in which

$$
g:=\theta_{\psi_{g}}, \quad h:=\theta_{\psi_{h}}
$$

are induced from characters $\psi_{g}$ and $\psi_{h}$ of (the same) mixed signature of a common real quadratic field $K$ is of special interest, since in that case $\varrho_{g h}$ factors through the Galois group of an abelian extension of $K$. For instance, when (31) holds, the representation $V_{g h}$ decomposes as a direct sum

$$
V_{g h}=V_{\psi^{+}} \oplus V_{\psi^{-}}, \quad \psi^{+}:=\psi_{g} \psi_{h}, \quad \psi^{-}:=\psi_{g} \psi_{h}^{\prime}
$$

of two induced representations by characters $\psi^{+}$and $\psi^{-}$of $K$ which are totally even and totally odd respectively.

If $V_{\psi^{+}}$is irreducible, it occurs with multiplicity two in the unit group, and hence $d_{g h}=2$. If $p$ is any prime satisfying the regularity hypothesis for $g$, then necessarily $p$ is inert in $K$ and therefore $\alpha_{g}=-\beta_{g}$, and likewise for $\alpha_{h}$ and $\beta_{h}$. The period $\Omega_{g h}$ of Conjecture 1.2 is predicted to be non-zero precisely when $\alpha_{g} \alpha_{h} \neq \pm 1$ and $\alpha_{g} \beta_{h} \neq \pm 1$. This period then involves, in its numerator, a $p$-adic regulator formed from genuine units in the $\psi^{+}$and $\left(\psi^{+}\right)^{\prime}$ components of the unit group of an appropriate abelian extension of $K$. The unit $u_{g_{\alpha}}$ 
which appears in the denominator in the definition of $\Omega_{g h}$ is just the $p$-adic logarithm of a fundamental unit $u_{K}$ of $K$, in light of the regularity hypothesis which implies that the prime $p$ is inert in $K$ (Cf. case (b) in the proof of Lemma 1.5 in Sec. 1.2 of [11]). We have not tested any instance of this general conjecture numerically, and it might be interesting to do so.

In the special case where $\psi_{h}=\psi_{g}^{-1}$, the character $\psi^{+}$is trivial and $\psi^{-}=\psi_{g} / \psi_{g}^{\prime}$ is a ring class character of $K$. The abelian extension $H$ of $K$ cut out by $\psi^{-}$is Galois over $\mathbb{Q}$ and $\mathrm{Gal}(H / \mathbb{Q})$ is a generalised dihedral group. The regularity hypothesis for $p$, which forces $p$ to be inert in $K / \mathbb{Q}$, implies that the frobenius element at $p$ acts on $V_{\psi^{-}}$with eigenvalues 1 and -1 , and hence the Artin representation $V_{\psi^{-}}$occurs with multiplicity 1 in the space $\mathbb{C} \otimes \mathcal{O}_{H}[1 / p]^{\times}$. The $p$-units in this abelian extension are precisely the Gross-Stark units studied in [8], and the regulator of Conjecture 1.2 involves the $p$-adic logarithms of such units. There is also the $p$-adic logarithm of a fundamental unit of $K$, which is in the minus eigenspace for the action of the frobenius automorphism at $p$, and which appears in both the numerator and the denominator and thus cancels out. The transcendental part of the period $\Omega_{g h}$ therefore involves only the $p$-adic logarithms of a $p$-unit $u_{p}^{+}\left(\psi^{-}\right)$, which is in the

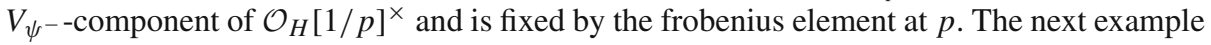
illustrates this special instance of Conjecture 1.2:

Example 5.1 Let $K=\mathbb{Q}(\sqrt{5})$ be the real quadratic field attached to the even Dirichlet character $\chi_{5}$ of conductor 5 , and let $\psi$ be a quartic character of $K$ whose conductor is one of the primes of $K$ above 29. The central character $\chi_{29}$ of $\psi$ is an odd quartic Dirichlet character of conductor 29 . Let $\chi:=\chi_{5} \chi_{29}$, and let

$$
g:=\theta_{\psi}=q-i q^{4}+i q^{5}-i q^{9}+(i-1) q^{11}-q^{16}+(i-1) q^{19}+\cdots \in S_{1}(5 \cdot 29, \chi)
$$

be the weight one modular form considered in [11, Example 4.1], whose Galois representation agrees with the induced representation $V_{\psi}$. After setting $h:=g^{*}=\theta_{\psi^{-1}}$, we have

$$
V_{g h}=1 \oplus \chi_{5} \oplus V_{\psi^{-}},
$$

where $\psi^{-}=\psi / \psi^{\prime}$ is a quartic ring class character of $\mathbb{Q}(\sqrt{5})$. The induced representation $V_{\psi^{-}}$has image the dihedral group $D_{4}$ of order 8 , and is notable in that it cannot be obtained as the induced representation of the character of any imaginary quadratic field.

Set $p:=13$ and choose the embedding of $\mathbb{Q}(i)$ into $\mathbb{Q}_{13}$ sending $i$ to the unique 4 th root of -1 which is congruent to 5 modulo 13 . One finds that $\chi(13)=1$ and $\left(\alpha_{g}, \beta_{g}\right)=(i,-i)$. Letting $f:=E_{2}^{(13)}(1,1)$, one computes

$$
\int_{\gamma_{ \pm i}} E_{2}^{(13)}(1,1) \cdot \theta_{\psi^{-1}}=-7724370085026350688358 \quad\left(\bmod 13^{20}\right) .
$$

Let

$$
H:=\mathbb{Q}(a) \quad \text { where } a^{8}-2 a^{7}+4 a^{6}-26 a^{5}+94 a^{4}-212 a^{3}+761 a^{2}-700 a+980 .
$$

This is the quartic subfield of the ring class field of $K$ of conductor 29, which is cut out by $\psi^{-}$. Let

$b:=\left(-27 a^{7}+116 a^{6}-196 a^{5}+738 a^{4}-3602 a^{3}+11256 a^{2}-22295 a+53310\right) / 5160$, 
denote the 13-unit of $H$ satisfying the quartic polynomial $x^{4}-11 x^{3}+20 x^{2}+89 x+169$. Writing Gal $(H / K)=\langle\sigma\rangle$, and defining $u_{13}^{+}\left(\psi^{-}\right):=b / b^{\sigma^{2}}$, we have verified that

$$
\int_{\gamma_{ \pm i}} E_{2}^{(13)}(1,1) \cdot \theta_{\psi^{-1}}=\frac{3}{2 \cdot 13} \cdot \log _{13}\left(u_{13}^{+}\left(\psi^{-}\right)\right),
$$

to 20-digits of 13-adic precision.

The setting in which

$$
g:=\theta_{\psi_{g}}, \quad h:=E_{1}\left(1, \chi_{g}^{-1}\right)
$$

is also of great relevance for explicit class field theory for $K$, since in that case

$$
V_{g h}=V_{\psi_{g}} \oplus V_{\psi_{g}^{-1}}=V_{g} \oplus V_{g^{*}}
$$

Let $u\left(\psi_{g}\right), u\left(\psi_{g}^{\prime}\right), u\left(\psi_{g}^{-1}\right)$, and $u\left(\psi_{g}^{\prime}\right)$ denote the generators in $L \otimes \mathcal{O}_{H_{g}}^{\times}$for the relevant eigencomponent relative to the action of $\mathrm{Gal}\left(H_{g} / K\right)$. Since the prime $p$ is inert in $K$, these four units can be chosen in such a way that

$$
\sigma_{p} u\left(\psi_{g}\right)=u\left(\psi_{g}^{\prime}\right), \quad \sigma_{p} u\left(\psi_{g}^{-1}\right)=u\left(\psi_{g}^{\prime-1}\right) .
$$

Example 5.2 Let $g$ be the cusp form in level 145 from Example 5.1 but now take

$$
h:=E_{1}(1, \chi)=(i+1)+q+\cdots+2 q^{13}+\cdots
$$

to be the Eisenstein series of weight 1 attached to the pair of characters $(1, \chi)$. The Artin representation $\varrho_{g h}$ is then given by

$$
V_{g h}=V_{\psi_{g}} \oplus V_{\psi_{g}^{-1}}=V_{g} \oplus V_{g^{*}}
$$

The smallest abelian extension $H_{g}$ of $K$ cut out by the representation $V_{g}$ (and/or $V_{g^{*}}$ ) is larger than the ring class field $H$ of degree 8 over $\mathbb{Q}$ that arose in Example 5.1: it is the ray class field of $K$ of conductor

$$
(29)=(29,2 \sqrt{5}+17)(29,2 \sqrt{5}+10)=: Q_{1} Q_{2},
$$

and has degree 32 over $\mathbb{Q}$. The field $H_{g}=H_{g^{*}}$ is the compositum of the ray class fields $H_{1}$ and $H_{2}$ of $K$ of conductors $Q_{1}$ and $Q_{2}$, these ray class fields being the abelian extensions of $K$ cut out by the characters $\psi_{g}$ and $\psi_{g}^{\prime}$ respectively.

A calculation with Magma reveals that $H_{g}$ can be described as the splitting field of the polynomial

$$
x^{8}-x^{7}-3 x^{6}+3 x^{5}+3 x^{4}-6 x^{3}-2 x^{2}+3 x+1=P_{1}(x) P_{2}(x)
$$

where

$$
P_{1}(x)=x^{4}+\frac{(-\sqrt{5}-1)}{2} x^{3}-x^{2}+x+\frac{(-\sqrt{5}+3)}{2}, \quad P_{2}(x)=\tau P_{1}(x),
$$

and $\tau$ denotes the generator of $\mathrm{Gal}(K / \mathbb{Q})$. The ray class fields $H_{1}$ and $H_{2}$ are just the splitting fields over $K$ of the irreducible quartic factors $P_{1}(x)$ and $P_{2}(x)$ above with cyclic Galois group. Hence, $H_{1}=K\left(u_{1}\right)$ and $H_{2}=K\left(u_{2}\right)$, where $u_{1}$ and $u_{2}$ are roots of $P_{1}(x)$ and $P_{2}(x)$ respectively. We define

$$
v_{1}:=\frac{1}{2}(-\sqrt{5}-1) u_{1}^{2}, \quad v_{2}:=\frac{1}{2}(\sqrt{5}-1) u_{2}^{2}
$$


Letting $\sigma_{1}$ denote a generator of $\mathrm{Gal}\left(H_{1} / K\right)$, we have

$$
v_{1}=\left(\sigma_{1}^{2}\left(v_{1}\right)\right)^{-1}, \quad \sigma_{1}\left(v_{1}\right)=\left(\sigma_{1}^{3}\left(v_{1}\right)\right)^{-1},
$$

and the unit $v_{1}$ and its $\mathrm{Gal}\left(H_{1} / K\right)$-translates $\sigma_{1} v_{1}, \sigma_{1}^{2} v_{1}, \sigma_{1}^{3} v_{1}$ generates the compositum of the $\psi_{g}$ and $\psi_{g}^{-1}$-isotypic parts in the unit group $\mathcal{O}_{H_{1}}^{\times}$. More precisely, using additive notation in $L \otimes \mathcal{O}_{H_{g}}^{\times}$, the elements

$$
v\left(\psi_{g}\right):=v_{1}+i \otimes v_{1}^{\sigma_{1}}, \quad v\left(\psi_{g}^{\prime}\right):=v_{2}+i \otimes v_{2}^{\sigma_{2}}
$$

generate the $\psi_{g}$ and $\psi_{g}^{\prime}$ eigenspaces respectively for the action of $\mathrm{Gal}\left(H_{g} / K\right)$, while the elements

$$
v\left(\psi_{g}^{-1}\right):=v_{1}-i \otimes v_{1}^{\sigma_{1}}, \quad v\left(\left(\psi_{g}^{\prime}\right)^{-1}\right):=v_{2}-i \otimes v_{2}^{\sigma_{2}}
$$

generate the $\psi_{g}^{-1}$ and $\left(\psi_{g}^{\prime}\right)^{-1}$-eigenspaces for this action.

Assume that $p$ is inert in $K$ and that $\alpha_{g}=-\beta_{g}=i$. After fixing $v_{1}$ and $v_{2}$ in such a way that $\sigma_{p}\left(v_{1}\right)=v_{2}$, a direct calculation reveals that the regulator matrix $R_{g_{i}}\left(\varrho_{g h}\right)$ is a diagonal matrix of the form

$$
R_{g_{i}}\left(\varrho_{g h}\right)=\left(\begin{array}{cc}
\log _{p}\left(v\left(\psi_{g}\right)\right)+i \log _{p}\left(v\left(\psi_{g}^{\prime}\right)\right) & 0 \\
0 & \log _{p}\left(v\left(\psi_{g}^{-1}\right)\right)+i \log _{p}\left(v\left(\left(\psi_{g}^{\prime}\right)^{-1}\right)\right)
\end{array}\right),
$$

while

$$
R_{g_{-i}}\left(\varrho_{g h}\right)=\left(\begin{array}{cc}
\log _{p}\left(v\left(\psi_{g}\right)\right)-i \log _{p}\left(v\left(\psi_{g}^{\prime}\right)\right) & 0 \\
0 & \log _{p}\left(v\left(\psi_{g}^{-1}\right)\right)-i \log _{p}\left(v\left(\left(\psi_{g}^{\prime}\right)^{-1}\right)\right)
\end{array}\right) .
$$

Let $p:=13$ and embed $\mathbb{Q}(i)$ into $\mathbb{Q}_{p}$ so that $i \equiv 5 \bmod p$. A numerical calculation of the $p$-adic iterated integrals reveals that

$$
\begin{aligned}
& \int_{\gamma_{+i}} E_{2}^{(13)}(1,1) \cdot E_{1}(1, \chi)=-8501755713365462060121 \quad\left(\bmod 13^{20}\right) \\
& \int_{\gamma_{-i}} E_{2}^{(13)}(1,1) \cdot E_{1}(1, \chi)=4274893846350140558653 \quad\left(\bmod 13^{20}\right) .
\end{aligned}
$$

After further embedding $H_{1}$ and $H_{2}$ into $\mathbb{Q}_{p^{4}}$ in such a way that $\sigma_{p}\left(v_{1}\right)=v_{2}$, one finds that

$$
\begin{aligned}
\int_{\gamma_{i}} E_{2}^{(13)}(1,1) \cdot h & =\frac{(7 i+6)^{2} \cdot \operatorname{det} R_{g_{i}}\left(\varrho_{g h}\right)}{3 \cdot 8 \cdot 7 \cdot 13 \cdot \log _{13}\left(u_{K}\right)} \\
\int_{\gamma_{-i}} E_{2}^{(13)}(1,1) \cdot h & =\frac{(-7 i+6)^{2} \cdot \operatorname{det} R_{g_{-i}}\left(\varrho_{g h}\right)}{3 \cdot 8 \cdot 7 \cdot 13 \cdot \log _{13}\left(u_{K}\right)},
\end{aligned}
$$

to 20 -digits of 13 -adic precision.

The analogous setting to that in Example 5.2 with Mordell-Weil groups replacing groups of units was discussed in [11, Section 6]. However, this case of the "Elliptic Stark Conjecture" was difficult to check experimentally because of the apparent scarcity of elliptic curves $E$ for which $r\left(E, \varrho_{g}\right)>0$, and no evidence was given in [11] (beyond the case when $g=g^{*}$ ). Example 5.2 can be seen as providing some indirect evidence for the main conjecture of [11], albeit with unit groups replacing Mordell-Weil groups. 


\section{Exotic forms}

Cases where $g$ and/or $h$ are exotic forms often correspond to settings that have been little studied previously from the point of view of either the classical or the $p$-adic Stark conjectures.

One of the simplest settings occurs when $g$ is exotic and $h=g^{*}$ is the dual form. The Artin representation $\varrho_{g h}$ then factors through the extension $H$ cut out by the projective representation attached to $g$, and it decomposes as

$$
V_{g h}=1 \oplus \operatorname{Ad}^{0}\left(V_{g}\right) .
$$

The adjoint representation $\operatorname{Ad}^{0}\left(V_{g}\right)$ always occurs with multiplicity one in $\mathbb{C} \otimes \mathcal{O}_{H}^{\times}$, and with multiplicity at least two in $\mathbb{C} \otimes \mathcal{O}_{H}[1 / p]^{\times}$, since 1 always arises as an eigenvalue for any linear transformation on the adjoint. More precisely, the frobenius automorphism $\sigma_{p}$ acts on $\operatorname{Ad}^{0}\left(V_{g}\right)$ with eigenvalues $1, \alpha_{g} / \beta_{g}$ and $\beta_{g} / \alpha_{g}$. When $\operatorname{Ad}^{0}\left(V_{g}\right)$ is regular at $p$, there are therefore (unique up to scaling) elements

$$
u_{1}, u_{g_{\alpha}}, u_{g_{\beta}} \in L \otimes \mathcal{O}_{H}^{\times}
$$

generating a subrepresentation of $L \otimes \mathcal{O}_{H}^{\times}$which is isomorphic to $\operatorname{Ad}^{0}\left(V_{g}\right)$, and such that

$$
\sigma_{p} u_{1}=u_{1}, \quad \sigma_{p} u_{g_{\alpha}}=\left(\alpha_{g} / \beta_{g}\right) \otimes u_{g_{\alpha}}, \quad \sigma_{p} u_{g_{\beta}}=\left(\beta_{g} / \alpha_{g}\right) \otimes u_{g_{\beta}} .
$$

Likewise, the choice of a Galois-equivariant homomorphism $v: V_{g h} \longrightarrow L \otimes\left(\mathcal{O}_{H}[1 / p]\right)^{\times}$ whose image is not contained in $L \otimes \mathcal{O}_{H}^{\times}$produces three independent $p$-units

$$
v_{1}, v_{g_{\alpha}}, v_{g_{\beta}} \in L \otimes\left(\mathcal{O}_{H}[1 / p]\right)^{\times}
$$

satisfying

$$
\sigma_{p} v_{1}=v_{1}, \quad \sigma_{p} v_{g_{\alpha}}=\left(\alpha_{g} / \beta_{g}\right) \otimes v_{g_{\alpha}}, \quad \sigma_{p} v_{g_{\beta}}=\left(\beta_{g} / \alpha_{g}\right) \otimes v_{g_{\beta}} .
$$

These units are the counterparts of the Stark points considered in [11, Example 5.3].

A simple calculation reveals that

$$
R_{g_{\alpha}}\left(\varrho_{g h}\right)=\left(\begin{array}{ll}
\log _{p} u_{1} & \log _{p} u_{g_{\alpha}} \\
\log _{p} v_{1} & \log _{p} v_{g_{\alpha}}
\end{array}\right),
$$

and therefore that the period $\Omega_{g h}$ of Conjecture 1.2 can be described in this case by

$$
\Omega_{g h}=\frac{\log _{p} u_{1} \log _{p} v_{g_{\alpha}}-\log _{p} u_{g_{\alpha}} \log _{p} v_{1}}{\log _{p} u_{g_{\alpha}}} .
$$

Example 6.1 This example examines this special case of Conjecture 1.2 when $g$ is Tate's tetrahedral form in level 133. Recall the following from [11, Example 5.3]: Let $\chi$ be a Dirichlet character of level $133=7 \cdot 19$ of order 2 at 7 and order 3 at 19 . Denote by $\zeta$ a primitive 12-th root of unity and define $\zeta_{3}:=\zeta^{-4}$. Then $S_{1}(133, \chi)$ is defined over $\mathbb{Q}\left(\zeta_{3}\right)$ and has dimension two. It contains two eigenforms defined over $\mathbb{Q}(\zeta)$, namely

$$
\begin{aligned}
h= & q+\left(\zeta^{2}-1\right) q^{2}-\zeta q^{3}+\zeta q^{5}+\left(-\zeta^{3}+\zeta\right) q^{6} \\
& +\zeta^{3} q^{7}-q^{8}+\left(\zeta^{3}-\zeta\right) q^{10}+\left(-\zeta^{3}+\zeta\right) q^{13}-\cdots
\end{aligned}
$$

and its Galois conjugate obtained by replacing $\zeta$ by $-\zeta$. Let $g=h^{*}$, the twist of $h$ by $\chi^{-1}$. The extension $H$ of $\mathbb{Q}$ cut out by the projective representation attached to $g$ is the normal closure of the quartic field 


$$
M:=\mathbb{Q}(w), \quad w^{4}+3 w^{2}-7 w+4=0,
$$

which has discriminant $133^{2}$ and Galois group $A_{4}$ over $\mathbb{Q}$.

As in [11, Example 5.3], with $p:=13$ we find the two 13-stabilisations $g_{\alpha}$ and $g_{\beta}$ of $g$ have $U_{p}$-eigenvalues $\alpha_{g}=\zeta^{-1}$ and $\beta_{g}=\zeta^{3}$, and choose the embedding of $\mathbb{Q}(\zeta)$ into $\mathbb{Q}_{13}$ which sends $\zeta$ to the 12th root of unity which is congruent to 6 modulo 13 .

Letting $f:=E_{2}^{(13)}(1,1)$ be the Eisenstein series of weight 2 and level 13, and letting $\gamma_{\alpha}$ be the element of the dual space of $S_{2}(133 \cdot p, \chi)\left[g_{\alpha}\right]$ sending $g_{\alpha}$ to 1 , (and likewise for $\gamma_{\beta}$, with $g_{\alpha}$ replaced by $g_{\beta}$ ), we find

$$
\begin{aligned}
& \int_{\gamma_{\alpha}} E_{2}^{(13)}(1,1) \cdot g=-5574982888910767132405 \quad\left(\bmod 13^{20}\right) \\
& \int_{\gamma_{\beta}} E_{2}^{(13)}(1,1) \cdot g=7956424094204877500900 \quad\left(\bmod 13^{20}\right) .
\end{aligned}
$$

Define the unit and 13-unit

$$
\begin{aligned}
u & :=-b+1 \in \mathcal{O}_{M}^{\times} \\
v & :=6 b^{3}+3 b^{2}+22 b-21 \in \mathcal{O}_{M}[1 / p]^{\times},
\end{aligned}
$$

respectively. One may choose an embedding of $H$ into $\mathbb{Q}_{13^{3}}$ so that $u_{1}:=u$ and $v_{1}:=v$ lie in $\mathbb{Q}_{13}$ and so are fixed by $\sigma_{13}$. Let

$$
u_{g_{\alpha}}, u_{g_{\beta}} \in\left(\mathcal{O}_{H}^{\times}\right)_{L}^{\mathrm{Ad}_{g}}, \quad v_{g_{\alpha}}, v_{g_{\beta}} \in\left(\mathcal{O}_{H}[1 / p]^{\times}\right)_{L}^{\operatorname{Ad}_{g}}
$$

be the units and $p$-units described in terms of $u$ and $v$ in (33) and (34). The unit $u_{\alpha_{g}}$ (resp. $u_{\beta_{g}}$ ) lies in the $\alpha_{g} / \beta_{g}=\zeta_{3}$ eigenspace (resp. the $\beta_{g} / \alpha_{g}=\zeta_{3}^{-1}$ eigenspace) for $\sigma_{p}$, and likewise for the $p$-units $v_{\alpha_{g}}$ and $v_{\beta_{g}}$.

To 20-digits of 13 -adic precision we have found

$$
\int_{\gamma_{\alpha}} E_{2}^{(13)}\left(1, \chi^{-1}\right) \cdot g=-\frac{\log _{p}\left(u_{1}\right) \log _{p}\left(v_{g_{\alpha}}\right)-\log _{p}\left(v_{1}\right) \log _{p}\left(u_{g_{\alpha}}\right)}{13 \cdot \log _{p}\left(u_{g_{\alpha}}\right)}
$$

and likewise replacing $\alpha$ by $\beta$ throughout.

This example is of a similar flavour to [11, Example 5.4, curves 26b, 52a]. Indeed, it pre-dates the rank 2 examples in [11] and was the experiment which led to the first serious evidence for (and correct formulation of) the "Elliptic Stark conjecture" of [11] in the more striking, but harder to test scenarios of "genuine rank two".

Our final example involves a scenario where $V_{g h}$ is an irreducible 4-dimensional representation, occuring with multiplicity two in the group of $p$-units. It is worth noting that no instances of this sort were ever successfuly tested numerically in the elliptic curve setting considered in [11], owing to the apparent scarcity of irreducible $V_{g h}$ of manageable size occuring with multiplicity two in the Mordell-Weil groups of elliptic curves of reasonable conductor. In the setting of units, such examples abound, since any irreducible $V_{g h}$ has multiplicity two in the unit group. The experiment below can thus also be viewed as providing some indirect support for the elliptic Stark conjecture of [11] in the numerically less accessible setting where $\varrho_{g h}$ is irreducible.

Example 6.2 Let $\chi$ be the quadratic Dirichlet character of level 283. The space $S_{1}(283, \chi)$ is 3-dimensional and spanned by the eigenforms 


$$
\begin{aligned}
g= & q+q^{4}-q^{7}+q^{9}-q^{11}-q^{13}+q^{16}-q^{23}+\cdots \\
h_{ \pm}= & q \pm \sqrt{-2} q^{2} \mp \sqrt{-2} q^{3}-q^{4} \mp \sqrt{-2} q^{5} \\
& +2 q^{6}-q^{7}-q^{9}+2 q^{10}+q^{11} \pm \sqrt{-2} q^{12}+\cdots .
\end{aligned}
$$

The pair $h_{ \pm}$are exotic forms whose associated projective representations have image $S_{4}$, and $g$ is the dihedral form who representation $\varrho_{g}$ is induced from an unramified character of the imaginary quadratic field $\mathbb{Q}(\sqrt{-283})$ (which has class number 3). The representation $\varrho_{g}$ is obtained by composing the projective representation attached to $\varrho_{h}$ with the exceptional homomorphism $S_{4} \longrightarrow S_{3}$. This is precisely the set-up of Lemma 2.3, which asserts that $\varrho_{g h}$ is irreducible and induced from a suitable quadratic character $\eta$ of a quartic extension $K$ of $\mathbb{Q}$. The quartic field $K$ occuring in this lemma, whose Galois closure $\bar{H}_{h}$ is the $S_{4}$-extension cut out by the projective representation attached to the exotic forms $h_{ \pm}$, is equal in this case to

$$
K=\mathbb{Q}(a), \quad a^{4}-a-1=0 .
$$

The quadratic extension $M$ of $K$ cut out by $\eta$ is given by

$$
M:=K(b) \quad \text { where } b^{2}=-6 a^{3}+a^{2}-3 a+5 .
$$

The elements

$$
\begin{aligned}
& u:=\frac{1}{2}\left(-a^{3}+a^{2}-a+1\right) b+\frac{1}{2}\left(-4 a^{3}+3 a^{2}-2 a+4\right) \\
& v:=\frac{1}{2}\left(a^{3}-2 a\right) b+\frac{1}{2}\left(a^{2}+2 a-3\right)
\end{aligned}
$$

are relative units in $M / K$, and hence their Galois translates generate the $\varrho_{g} h$-isotypic components of the unit group of $H_{h}$. In additive notation let

$$
u^{\zeta_{8}^{ \pm 1}}:=\sum_{j=0}^{7} \zeta_{8}^{\mp j} u^{\sigma_{p}^{j}}
$$

and likewise for $v^{ \pm \zeta_{8}}$. Here $\zeta_{8}$ is a primitive 8 th root of unity such that $\sqrt{-2}=\zeta_{8}^{3}+\zeta_{8}$.

Let $p:=5$, so that $\chi(5)=-1$ and $\alpha_{g}=-\beta_{g}=1$. Taking $f=E_{2}^{(5)}(1,1)$, one computes

$$
\begin{aligned}
& \int_{\gamma_{+1}} E_{2}^{(5)}(1,1) \cdot h_{ \pm} \\
& =(1365364418900524107116682 \pm 982832041040545110371696 \sqrt{-2}) / 5
\end{aligned}
$$

to 35-digits of 5-adic precision (and likewise for the -1 -stabilisation, replacing \pm on the righthand side by $\mp$ ), after choosing an embedding of $\mathbb{Q}\left(\zeta_{8}\right)$ and $H$ into $\overline{\mathbb{Q}_{p}}$. A direct calculation shows that in this example,

$$
\operatorname{det} R_{g_{\alpha}}\left(\varrho_{g h}\right)=\log _{p}\left(u^{\zeta 8}\right) \log _{p}\left(v^{\zeta_{8}^{-1}}\right)-\log _{p}\left(u^{\zeta_{8}^{-1}}\right) \log _{p}\left(v^{\zeta 8}\right) .
$$

As for the Stark unit $u_{g_{\alpha}}$ occuring in the denominator of the period $\Omega_{g h}$ of Conjecture 1.2 when $\alpha_{g}=-1$, the Hilbert class field of $\mathbb{Q}(\sqrt{-283})$ is generated by an algebraic number $w$ satisfying the relation $w^{3}-16 w^{2}-8 w-1=0$. The unit $u_{g_{\alpha}}$ is then described, using additive notation, by the formula

$$
u_{g_{\alpha}}:=w^{\sigma_{p}}-w^{\sigma_{p}^{2}}
$$


where an embedding of the Hilbert class field into $\mathbb{Q}_{p^{2}}$ has been fixed so that $w$ is defined over $\mathbb{Q}_{p}$. It has been verified experimentally that

$\int_{\gamma_{+1}} E_{2}^{(5)}(1,1) \cdot h_{-}=\frac{19 \zeta_{8}^{3}+34 \zeta_{8}^{2}-19 \zeta_{8}}{2^{5} \cdot 3^{2} \cdot 5} \cdot \frac{\log _{5}\left(u^{\zeta_{8}}\right) \log _{5}\left(v^{\zeta_{8}^{-1}}\right)-\log _{5}\left(u^{\zeta_{8}^{-1}}\right) \log _{5}\left(v^{\zeta_{8}}\right)}{\log _{5}\left(u_{g_{\alpha}}\right)}$

to 35 digits of 5-adic precision, in excellent agreement with Conjecture 1.2.

Acknowledgments The first author was supported by an NSERC Discovery grant, and the third by Grant MTM2012-34611.

\section{References}

1. Bertolini, M., Castella, F., Darmon, H., Dasgupta, S., Prasanna, K., Rotger, V.: p-functions and Euler systems: a tale in two trilogies. In: Proceedings of the 2011 Durham symposium on Automorphic forms and Galois representations. London Math. Society Lecture Notes, vol. 414, pp. 52-101 (2014)

2. Bertolini, M., Darmon, H.: Kato's Euler system and rational points on elliptic curves I: a p-adic Beilinson formula. Israel J. Math. 199, 163-188 (2014)

3. Bertolini, M., Darmon, H., Rotger, V.: Beilinson-Flach elements and Euler systems I: syntomic regulators and $p$-series. J. Algebraic Geom. 24, 355-378 (2015)

4. Bertolini, M., Darmon, H., Rotger, V.: Beilinson-Flach elements and Euler systems II: the Birch and Swinnerton-Dyer conjecture for Hasse-Weil-Artin L-series. J. Algebraic Geom. 24, 569-604 (2015)

5. Bellaïche, J., Dimitrov, M.: On the eigencurve at classical weight one points, accepted to Duke Math. J

6. Charollois, P., Darmon, H.: Arguments des unités de Stark et périodes de séries d'Eisenstein. Algebra Number Theory 2(6), 655-688 (2008)

7. Darmon, H.: Integration on $\mathcal{H}_{p} \times \mathcal{H}$ and arithmetic applications. Ann. Math. (2) 154(3), 589-639 (2001)

8. Darmon, H., Dasgupta, S.: Elliptic units for real quadratic fields. Ann. Math. (2) 163(1), 301-346 (2006)

9. de Shalit, E.: Iwasawa theory of elliptic curves with complex multiplication. $p$-functions. In: Perspectives in Mathematics, vol. 3. Academic Press, Inc., Boston (1987)

10. Darmon, H., Logan, A.: Periods of Hilbert modular forms and rational points on elliptic curves. Int. Math. Res. Not. 40, 2153-2180 (2003)

11. Darmon, H., Lauder, A., Rotger, V.: Stark points and $p$-adic iterated integrals attached to modular forms of weight one, Forum. Math., Pi, vol. 3, e8, 95 (2015)

12. Darmon, H., Pollack, R.: Efficient calculation of Stark-Heegner points via overconvergent modular symbols. Israel J. Math. 153(1), 319-354 (2006)

13. Darmon, H., Rotger, V.: Diagonal cycles and Euler systems I: a p-adic Gross-Zagier formula. Annales Sc. Ecole Norm. Supérieure 47(4), 779-832 (2014)

14. Darmon, H., Rotger, V.: Diagonal cycles and Euler systems II: the Birch and Swinnerton-Dyer conjecture for Hasse-Weil-Artin $L$-series, submitted

15. Dasgupta, S.: Stark's conjectures. Senior Master Thesis, Harvard University (1999)

16. Deligne, P., Serre, J.-P.: Formes modulaires de poids 1. Ann. Sc. École N. Sup. 7, 507-530 (1974)

17. Gross, B.: On the factorization of $p$-adic $L$-series. Invent. Math. 57(1), 83-95 (1980)

18. Katz, N.M.: $p$-adic interpolation of real analytic Eisenstein series. Ann. Math. 104(3), 459-571 (1976)

19. Lauder, A.: Efficient computation of Rankin p-functions. In: Böckle, G., Wiese, G. (eds) Computations with Modular Forms, pp. 181-200. Springer-Verlag, Berlin (2014)

20. Serre, J.-P.: Modular forms of weight one and Galois representations. In: Algebraic number fields: $L$ functions and Galois properties (Proc. Sympos., Univ. Durham, Durham, 1975), pp. 193-286. Academic Press, London (1977)

21. Stark, H.M.: L-functions with Rational Characters. Advances Math., vol. 17, pp. 60-92 (1975)

22. Stevens, G.H.: The cuspidal group and special values of $L$-functions. Trans. Am. Math. Soc. 291(2), 519-550 (1985)

23. Stevens, G.H.: The Eisenstein Measure and Real Quadratic Fields. Théorie des nombres (Quebec, 1987), pp. 887-927. de Gruyter, Berlin (1989)

24. Stevens, G.H.: $K$-theory and Eisenstein series, preprint (2007)

25. Tate, J.: Les conjectures de Stark sur les Fonctions L. Progress in Math., vol. 47. Birkhäuser (1984) 